\title{
Coupling aerosol optics to the MATCH (v5.5.0) chemical transport model and the SALSA (v1) aerosol microphysics module
}

\author{
Emma Andersson $^{1}$ and Michael Kahnert ${ }^{1,2}$ \\ ${ }^{1}$ Department of Earth and Space Sciences, Chalmers University of Technology, 41296 Gothenburg, Sweden \\ ${ }^{2}$ Swedish Meteorological and Hydrological Institute, 60176 Norrköping, Sweden \\ Correspondence to: Michael Kahnert (michael.kahnert@ smhi.se)
}

Received: 3 November 2015 - Published in Geosci. Model Dev. Discuss.: 21 December 2015

Revised: 21 April 2016 - Accepted: 25 April 2016 - Published: 12 May 2016

\begin{abstract}
A new aerosol-optics model is implemented in which realistic morphologies and mixing states are assumed, especially for black carbon particles. The model includes both external and internal mixing of all chemical species, it treats externally mixed black carbon as fractal aggregates, and it accounts for inhomogeneous internal mixing of black carbon by use of a novel "core-grey-shell" model. Simulated results of aerosol optical properties, such as aerosol optical depth, backscattering coefficients and the Ångström exponent, as well as radiative fluxes are computed with the new optics model and compared with results from an older opticsmodel version that treats all particles as externally mixed homogeneous spheres. The results show that using a more detailed description of particle morphology and mixing state impacts the aerosol optical properties to a degree of the same order of magnitude as the effects of aerosol-microphysical processes. For instance, the aerosol optical depth computed for two cases in 2007 shows a relative difference between the two optics models that varies over the European region between -28 and $18 \%$, while the differences caused by the inclusion or omission of the aerosol-microphysical processes range from -50 to $37 \%$. This is an important finding, suggesting that a simple optics model coupled to a chemical transport model can introduce considerable errors affecting radiative fluxes in chemistry-climate models, compromising comparisons of model results with remote sensing observations of aerosols, and impeding the assimilation of satellite products for aerosols into chemical-transport models.
\end{abstract}

\section{Introduction}

Aerosol-optics models are employed in large-scale chemical transport models (CTMs) in mainly two contexts, namely, in chemistry-climate modelling (CCM), and in conjunction with remote sensing observations. In a CCM one couples a CTM to an atmosphere-ocean general circulation model (GCM). One purpose is to account for the dynamic effects of aerosol particles on cloud microphysics. Another is to obtain a better description of the direct effect of aerosol particles and radiatively active trace gases on the radiative balance. The aerosol-optics model provides a link that converts the aerosol fields delivered by the CTM to the aerosol optical properties that are required as input to the radiative transfer model, with which one computes the radiative energy budget. In remote sensing applications one is faced with the obstacle that the aerosol concentration fields computed with a CTM are not directly comparable to the radiometric quantities that are observed with remote sensing instruments. The aerosoloptics model provides the observation operator that maps the CTM output to radiometric variables that can be compared to satellite observations or satellite retrieval products. This allows us to either employ satellite observations for evaluating CTM model results, or to assimilate satellite data into a CTM-based air-quality forecasting system. It is clear that the aerosol-optics model has a pivotal role in these kinds of applications. It may constitute an additional source of error that could compromise the reliability of CCMs, impair the reliability of CTM evaluations, or degrade chemical data assimilation results. It is, therefore, important to better understand this potential source of error, quantify its possible impact on model predictions of aerosol radiometric quantities, 
and assess the level of morphological detail that might be required in aerosol-optics models coupled to CTMs.

A main difficulty is that aerosol particles in nature can have a high degree of morphological complexity. For instance, mineral dust particles can have irregular shapes, small-scale surface roughness, and inhomogeneous mineralogical composition (e.g. Nousiainen, 2009). Black carbon particles suspended in air have fractal-aggregate shapes (e.g. Jones, 2006) that can be coated by weakly absorbing liquid-phase components that condense onto the aggregates as they age in the atmosphere (e.g. Adachi and Buseck, 2008). Volcanic ash particles are composed of crustal material in which multiple air vesicles may have been trapped during the generation of the particles. In aerosol-optics models one has to make a choice what level of morphological detail is necessary and affordable. A detailed discussion of this question can be found in Kahnert et al. (2014).

In environmental modelling practical and computational constraints often force us to invoke drastically simplifying assumptions about aerosol morphology. For instance, one frequently computes aerosol optical properties based on the assumption that all chemical aerosol components are contained in separate particles (externally mixed), and that each such particle can be approximated as a homogeneous sphere. As pointed out in Kahnert (2008) and Benedetti et al. (2009), this approach is highly attractive from a practical point of view, because the aerosol optical observation operators, which map mixing ratios to radiometric properties, become linear functions of the mixing ratios of the different chemical species. A linearization of the observation operator is a prerequisite for most of the commonly used data-assimilation methodologies, such as the variational method (e.g. Kahnert, 2008; Benedetti et al., 2009). However, such approximations can also introduce substantial errors. In the remote sensing community awareness of this problem has been growing over the past 1-2 decades. As a result, one has developed retrieval methods for desert dust aerosol particles that are based on spheroidal model particles (e.g. Dubovik et al., 2006), which can mimic the optical properties of mineral dust particles better than homogeneous spheres (Kahnert, 2004; Nousiainen et al., 2006). In chemical data assimilation, the problem is still treated rather negligently. A few assimilation studies account for internal mixing (where several aerosol components can be contained within one particle) of different chemical components (e.g. Saide et al., 2013). But the particles are still assumed to be perfectly homogeneous spheres. To the best of our knowledge there are currently no aerosol optical observation operators in chemical transport models that take complex morphological properties of aerosol particles such as non-sphericity or inhomogeneous internal structure into account.

This study describes the coupling of two different aerosoloptics models to a regional CTM. One optics model is based on the simple external-mixture and homogeneous-sphere approximations. The second model takes both external and in- ternal mixing of aerosol components into account. Also, it employs morphologically more realistic models for black carbon particles. Although black carbon contributes, on average, only some $5 \%$ to the mass mixing ratio of particulate matter over Europe, it can have a significant global radiative warming effect. Previous theoretical studies on the optical properties of black carbon particles suggest that the use of homogeneous-sphere models can introduce substantial errors into the absorption cross section and single scattering albedo of such particles (e.g. Kahnert, 2010a; Kahnert et al., 2013). Also, the largest mixing-state sensitivity in both regional and global radiative fluxes comes from black carbon according to Klingmüller et al. (2014).

The main goal of this study is to assess the impact of aerosol morphology and mixing state on radiometric quantities and radiative forcing simulated with a chemical transport model. To this end we compare the two optics models, and we gauge the significance of morphology by comparing the differences in the optics model output to other sources of error. As a gauge we use the impact of including or omitting aerosol microphysical processes; this provides us with a reference which is generally agreed to have a significant effect on aerosol transport models (Andersson et al., 2015; Kokkola et al., 2008).

The CTM, its aerosol microphysic and mass transport setups, and the aerosol-optics models are described in Sect. 2. There we also explain the methodology we employ for comparison of the optics models. In Sect. 3 we present and discuss computational results for selected cases and for several radiative and optical parameters. Concluding remarks are given in Sect. 4.

\section{Model description and methods}

\subsection{General considerations and terminology}

Aerosol particles typically originate from different emission sources, such as sea-salt particles coming from marine sources, wind-blown dust from dry land surfaces, volcanic ash from magmatic or phreatomagmatic eruptions, or black carbon produced during combustion of fossil fuel, biofuel, or biomass. During atmospheric transport, particles from different sources can be mixed, resulting in heterogeneous aerosol populations consisting of particles of different morphologies, sizes, and chemical composition. A mixture in which different chemical species are contained in separate particles is referred to as an external mixture. On the other hand, aerosol dynamic processes, such as nucleation, condensation, and coagulation, give rise to the formation and growth of secondary particles from precursor gases, as well as to the condensation of precursor gases onto existing primary particles. These processes result in particles in which several chemical species are mixed with each other in one and the same particle. Such a population is referred to as an inter- 
nal mixture. There are two types of internal mixtures. If e.g. hydrophilic liquid-phase components mix with each other, one can obtain a homogeneous internal mixture of different chemical species. On the other hand, condensation of gasphase species onto non-soluble primary particles or cloud processing of aerosol particles can result in liquid-phase material coating a solid core of e.g. mineral dust or black carbon. We refer to the latter as an inhomogeneous internal mixture. Aerosol populations in nature are often both externally and internally mixed, i.e. they contain particles that are composed of a single chemical species as well as other particles that are composed of different chemical species, which can be homogeneously or inhomogeneously internally mixed.

Aerosol optical properties are strongly dependent on not only the size and chemical composition, but also on the mixing state, shape, and internal structure of particles. Therefore, before explaining the aerosol-optics model, we first need to briefly describe the kind of information that can be provided by the aerosol transport model. In particular, we need to understand the level of detail with which the size distribution, size-dependent chemical composition, and the mixing state of the aerosol particles can be computed in a large-scale model.

\subsection{Aerosol transport modelling with MATCH}

As a regional model we employ the Multiple-scale Atmospheric Transport and CHemistry modelling system (MATCH), an offline Eulerian model developed by the Swedish Meteorological and Hydrological Institute (Andersson et al., 2007). For this study we have set up the MATCH model over the European domain with a $0.4 \times 0.4^{\circ}$ horizontal resolution and a rotated latitude-longitude grid, covering about $34^{\circ}$ longitude and $42^{\circ}$ latitude. The model has 40 vertical $\eta$ layers with varying thicknesses depending on the topography, and it extends up to about $13 \mathrm{hPa}$. The meteorological input comes from the HIRLAM (HIgh-Resolution Limited Area Model) numerical weather-prediction model (Unden et al., 2002).

The MATCH model allows us to choose between two aerosol model versions, a simpler mass-transport model, and a more sophisticated aerosol dynamic transport model.

\subsubsection{Mass transport model}

A simple version of the MATCH CTM, which we refer to as the "mass-transport model", neglects all aerosol dynamic processes. It contains a photochemistry model that computes mass concentrations of secondary inorganic aerosols (SIAs), which are formed from precursor gases. The SIA fraction of aerosol particles consists of ammonium sulfate $\left(\left(\mathrm{NH}_{4}\right)_{2} \mathrm{SO}_{4}\right)$, ammonium nitrate $\left(\mathrm{NH}_{4} \mathrm{NO}_{3}\right)$, other particulate sulfates $\left(\mathrm{PSO}_{x}\right)$, and other particulate nitrates $\left(\mathrm{PNO}_{x}\right)$. The mass transport model furthermore contains a sea-salt module that computes $\mathrm{NaCl}$ emissions based on the param- eterizations described in Mårtensson et al. (2003) and Monahan et al. (1986). More details on the MATCH photochemistry model can be found in Robertson et al. (1999) and Andersson et al. (2007); the MATCH sea-salt model is described in Foltescu et al. (2005). The mass transport model also contains a simple wind-blown dust model and a module for transport of primary particulate matter (PPM), i.e. aerosol particles other than sea salt and wind-blown dust that are emitted as particles, rather than being formed from gas precursors. The size bins in the PPM model are flexible. In the current model set-up the sea-salt and PPM models were run for four size bins as shown in Table 1. We used gridded EMEP PPM emission data for the year 2007 in conjunction with black carbon (BC) and organic carbon (OC) emission data by Kupiainen and Klimont $(2004,2007)$. The latter provide $\mathrm{BC}$ and $\mathrm{OC}$ emissions per country and emission sector. We distributed these among the grid cells in the model domain according to the EMEP PPM gridded emissions. Thus, the $\mathrm{BC}$ and $\mathrm{OC}$ emissions vary among grid cells in accordance with the EMEP PPM emissions, while the sum of all $\mathrm{BC}$ and $\mathrm{OC}$ emissions over all grid cells per country and emission sector agrees with the corresponding $\mathrm{BC}$ and $\mathrm{OC}$ emissions, respectively, reported in Kupiainen and Klimont (2004, 2007). The remaining emissions (PPM-BC-OC) in each grid cell are interpreted as dust particles. The primaryparticle emissions are distributed among the size bins; during atmospheric transport they remain chemically and dynamically inert in the model. Thus no chemical transformation, mixing processes with other compounds, or other sizetransformation processes are included in the model. The SIA components are given as total mass concentrations without any information about their size distribution. In the optics model a fixed size distribution is assumed to assign the total SIA mass to the four size bins. Water adsorption by particles is computed in the optics model as described in Sect. 2.3.1.

\subsubsection{Aerosol microphysics module - SALSA}

A more realistic description of particles can be achieved by accounting for aerosol microphysical processes. To this end the Sectional Aerosol module for Large Scale Applications (SALSA) (Kokkola et al., 2008) has recently been coupled to the MATCH photochemistry model (Andersson et al., 2015). This model tracks mass concentrations of different species per size bin, and particle number concentrations. Thus, it provides size-dependent composition and mixing state of aerosol particles. The description of $\mathrm{PNO}_{x}$, wind-blown dust, and secondary organic aerosols (SOA) is still in an early development stage in MATCH-SALSA. In the current version, $\mathrm{PNO}_{x}$ is simply computed according to the same photochemistry scheme as in the mass-transport model, and the $\mathrm{PNO}_{x}$ mass is assigned to size bin 15 (see Table 2). Wind-blown dust and SOA are absent in the present model version. The size distributions for the emitted particles can be found in Table 4 and Fig. 6 of Andersson et al. (2013). 
Table 1. Size bins (characterized by the radius $r$ ) and chemical species in the MATCH mass transport model (Andersson et al., 2007). The labels " $p$ " and "s" refer to primary emitted particles and secondary particles generated from gas precursors.

\begin{tabular}{lrccccccccc}
\hline $\begin{array}{l}\text { Size } \\
\text { bin }\end{array}$ & $r(\mathrm{~nm})$ & $\mathrm{OC}$ & $\mathrm{BC}$ & Dust & $\begin{array}{c}\text { Other } \\
\mathrm{PPM}\end{array}$ & $\mathrm{NaCl}$ & $\left(\mathrm{NH}_{4}\right)_{2} \mathrm{SO}_{4}$ & $\mathrm{NH}_{4} \mathrm{NO}_{3}$ & $\begin{array}{c}\text { Other } \\
\mathrm{PSO}_{x}\end{array}$ & $\begin{array}{c}\text { Other } \\
\mathrm{PNO}_{x}\end{array}$ \\
\hline 1 & $10-50$ & $\mathrm{p}$ & $\mathrm{p}$ & & $\mathrm{p}$ & $\mathrm{p}$ & $\mathrm{s}$ & $\mathrm{s}$ & $\mathrm{s}$ & $\mathrm{s}$ \\
2 & $50-500$ & $\mathrm{p}$ & $\mathrm{p}$ & & $\mathrm{p}$ & $\mathrm{p}$ & $\mathrm{s}$ & $\mathrm{s}$ & $\mathrm{s}$ & $\mathrm{s}$ \\
3 & $500-1250$ & & & & & $\mathrm{p}$ & $\mathrm{s}$ & $\mathrm{s}$ & $\mathrm{s}$ & $\mathrm{s}$ \\
4 & $1250-5000$ & $\mathrm{p}$ & $\mathrm{p}$ & $\mathrm{p}$ & $\mathrm{p}$ & $\mathrm{p}$ & $\mathrm{s}$ & $\mathrm{s}$ & $\mathrm{s}$ & $\mathrm{s}$ \\
\hline
\end{tabular}

Table 2 shows the current model set-up with the number and range of the size bins. As is evident from this table, MATCH-SALSA accounts for both internally and externally mixed aerosol particles. In total, there are 20 different size bins in MATCH-SALSA, each one of them representing a particle size range (volume-equivalent radius, $r$ ), mixing state, and composition. Some size bins have the same size range, but different mixing states and/or compositions. For instance, size bins 12, 15, and 18 describe the same size range $(350-873 \mathrm{~nm})$, but different internal mixtures of various species. Similarly, bins 4 and 8 have the same size range (25-49 nm), but one describes an internal mixture, the other an external mixture of aerosol species.

As in the mass-transport model, "other PPM", i.e. primary particles other than $\mathrm{BC}$ and $\mathrm{OC}$, are interpreted as dust particles. Note that water is not directly calculated as a prognostic variable in MATCH-SALSA. Rather, it is a diagnostic variable computed in the MATCH-optics model as explained in Sect. 2.3.2. The table merely indicates which size bins are assumed in the optics model to be internally mixed with adsorbed water. A more detailed description of the MATCHSALSA model can be found in Andersson et al. (2015).

\subsection{Aerosol-optics modelling}

Aerosol-optics models coupled to a CTM have to make consistent use of the information provided by the CTM, while invoking assumptions on optically relevant parameters that are not provided by the CTM. The parameters that influence the particles' optical properties are

- the aerosol size distribution;

- the refractive index of the materials of which the aerosol particles are composed; and

- the morphology of the particles.

Morphology refers to both the overall shape of the particle, and, in case of inhomogeneously mixed particles, the variation of the refractive index inside the particle.

The information provided by the CTM depends on the level of detail in the process descriptions. In the MATCH mass transport model, we have size information for the primary particles, but only the total mass for secondary inorganic aerosols. Thus we have to invoke assumptions about the size distribution of these particles. The MATCH optics models in conjunction with the MATCH mass transport model assume that $10 \%$ of the SIA aerosol mass are in the smallest size bin (see Table 1), $60 \%$ in the second, $20 \%$ in the third, and $10 \%$ in the fourth size bin. Also, the mass transport model lacks any information about the mixing state of the particles. We therefore have to invoke appropriate assumptions on whether the aerosol particles are externally or internally mixed. Both the mass transport model and MATCH-SALSA lack information on whether the internally mixed particles are homogeneous or inhomogeneous. Also, neither model provides any information on the shape of the particles. The refractive indices of each chemical component in the aerosol phase and their spectral variation are listed in Appendix D. They can also be found in Fig. 4 in Kahnert (2010a). That reference also contains detailed information about the different literature sources from which the refractive indices are taken.

\subsubsection{Optics model for externally mixed aerosol particles}

The simplest conceivable optics model assumes that all particles are homogeneous spheres, and that all chemical species are each in separate particles, i.e. externally mixed. As explained in Kahnert (2008), the external-mixture assumption results in a linear relation between the mass mixing ratios and the optical properties. Owing to the linearity, this model is particularly attractive for data assimilation applications (e.g. Benedetti et al., 2009), which require linearized observation operators. However, this is also the crudest possible optics model, as it neglects both the effect of internal mixing and of particle morphology on optical properties.

The external-mixture model is implemented in the MATCH mass transport model, where it is primarily being used in the MATCH 3DVAR data assimilation system (Kahnert, 2008). Optical properties are pre-computed for 12 wavelength bands ranging from the UV-C to the mid-IR. Dust and black carbon are assumed to be hydrophobic, while sea salt, OC, and SIA components can each mix internally with water. The water volume fraction depends on temperature and humidity; it is computed by use of the parameterization given in Gerber (1985), which computes the particle's wet radius as a function of dry radius, relative humidity, and temperature. 
Table 2. Size bins and chemical species in the MATCH-SALSA aerosol microphysical transport model. An " $x$ " marks that the species is present in a particular size bin.

\begin{tabular}{|c|c|c|c|c|c|c|c|c|c|}
\hline $\begin{array}{l}\text { Size } \\
\text { bin }\end{array}$ & $r(\mathrm{~nm})$ & $\begin{array}{l}\text { Mixing } \\
\text { state }\end{array}$ & $\mathrm{OC}$ & $\mathrm{BC}$ & $\begin{array}{l}\text { Other } \\
\text { PPM }\end{array}$ & $\mathrm{NaCl}$ & $\mathrm{PSO}_{x}$ & $\mathrm{PNO}_{x}$ & $\mathrm{PNH}_{x}$ \\
\hline 1 & $1.5-3.8$ & Internal & $\mathrm{x}$ & & & & $\mathrm{x}$ & & $\mathrm{x}$ \\
\hline 2 & $3.8-9.8$ & Internal & $\mathrm{x}$ & & & & $\mathrm{x}$ & & $\mathrm{x}$ \\
\hline 3 & $9.8-25$ & Internal & $\mathrm{x}$ & & & & $\mathrm{x}$ & & $\mathrm{x}$ \\
\hline 4 & $25-49$ & Internal $+\mathrm{H}_{2} \mathrm{O}$ & $\mathrm{x}$ & $\mathrm{x}$ & $\mathrm{x}$ & $\mathrm{x}$ & $\mathrm{x}$ & & $\mathrm{x}$ \\
\hline 5 & $49-96$ & Internal $+\mathrm{H}_{2} \mathrm{O}$ & $\mathrm{x}$ & $\mathrm{x}$ & $\mathrm{x}$ & $\mathrm{x}$ & $\mathrm{x}$ & & $\mathrm{x}$ \\
\hline 6 & $96-187$ & Internal $+\mathrm{H}_{2} \mathrm{O}$ & $\mathrm{x}$ & $\mathrm{x}$ & $\mathrm{x}$ & $\mathrm{x}$ & $\mathrm{x}$ & & $\mathrm{x}$ \\
\hline 7 & $187-350$ & Internal $+\mathrm{H}_{2} \mathrm{O}$ & $\mathrm{x}$ & $\mathrm{x}$ & $\mathrm{x}$ & $\mathrm{x}$ & $\mathrm{x}$ & & $\mathrm{x}$ \\
\hline 8 & $25-49$ & External & $\mathrm{x}$ & $\mathrm{x}$ & & & $\mathrm{x}$ & & $\mathrm{x}$ \\
\hline 9 & 49-96 & External & $\mathrm{x}$ & $\mathrm{x}$ & & & $\mathrm{x}$ & & $\mathrm{x}$ \\
\hline 10 & $96-187$ & External & $\mathrm{x}$ & $\mathrm{x}$ & & & $\mathrm{x}$ & & $\mathrm{x}$ \\
\hline 11 & $187-350$ & External & $\mathrm{x}$ & $\mathrm{x}$ & $\mathrm{x}$ & & $\mathrm{x}$ & & $\mathrm{x}$ \\
\hline 12 & $350-873$ & $\mathrm{NaCl}+\mathrm{H}_{2} \mathrm{O}$ & & & & $\mathrm{x}$ & & & \\
\hline 13 & 873-2090 & $\mathrm{NaCl}+\mathrm{H}_{2} \mathrm{O}$ & & & & $\mathrm{x}$ & & & \\
\hline 14 & $2090-5000$ & $\mathrm{NaCl}+\mathrm{H}_{2} \mathrm{O}$ & & & & $\mathrm{x}$ & & & \\
\hline 15 & $350-873$ & Internal $+\mathrm{H}_{2} \mathrm{O}$ & $\mathrm{x}$ & $\mathrm{x}$ & $\mathrm{x}$ & & $\mathrm{x}$ & $\mathrm{x}$ & $\mathrm{x}$ \\
\hline 16 & 873-2090 & Internal $+\mathrm{H}_{2} \mathrm{O}$ & $\mathrm{x}$ & & $\mathrm{x}$ & & $\mathrm{x}$ & & $\mathrm{x}$ \\
\hline 17 & $2090-5000$ & Internal $+\mathrm{H}_{2} \mathrm{O}$ & $\mathrm{x}$ & & $\mathrm{x}$ & & $\mathrm{x}$ & & $\mathrm{x}$ \\
\hline 18 & $350-873$ & Internal $+\mathrm{H}_{2} \mathrm{O}$ & & & $\mathrm{x}$ & & $\mathrm{x}$ & & $\mathrm{x}$ \\
\hline 19 & 873-2090 & Internal $+\mathrm{H}_{2} \mathrm{O}$ & & & $\mathrm{x}$ & & $\mathrm{x}$ & & $\mathrm{x}$ \\
\hline 20 & $2090-5000$ & Internal $+\mathrm{H}_{2} \mathrm{O}$ & & & $\mathrm{x}$ & & $\mathrm{x}$ & & $\mathrm{x}$ \\
\hline
\end{tabular}

The aerosol-water mixture is assumed to be homogeneous. The dielectric properties of a homogeneous mixture of two or more components are described by a complex effective refractive index $m_{\text {eff }}$, which is usually computed by effective medium theory (EMT) (although chemical transport modellers often use simple volume mixing rules, most likely because EMTs are not commonly known in that field). We use Bruggemann's EMT (Bruggemann, 1935). More information on EMT is given in Appendix C. Optical properties are precomputed for 11 water volume fractions between 0 and 0.98 ; for intermediate volume fractions the optical properties are linearly interpolated. The optical properties contained in the database are the extinction cross section $C_{\text {ext }}$, the scattering cross section $C_{\text {sca }}$, the value of the phase function in the exact backscattering direction $p\left(180^{\circ}\right)$, and the asymmetry parameter $g$.

As explained in Kahnert (2008), size-averaged optical properties are pre-computed by averaging over a log-normal size distribution $n_{i}(r)=$ $N_{i}^{0} /\left(\sqrt{2 \pi} r \ln \sigma_{i}\right) \exp \left[-\ln ^{2}\left(r / R_{i}\right) /\left(2 \ln ^{2} \sigma_{i}\right)\right]$ for each size bin $i$, where $N_{i}^{0}$ relates to the number density of particles in size bin $i, r$ denotes the particle radius, $R_{1}=0.022 \mu \mathrm{m}$, $R_{2}=0.158 \mu \mathrm{m}, R_{3}=0.791 \mu \mathrm{m}, R_{4}=2.5 \mu \mathrm{m}$ are the geometric mean radii in each size mode, and the geometric standard deviation $\sigma_{1}=\sigma_{3}=\sigma_{4}=1.8, \sigma_{2}=1.5$ are based on measurements in Neusüß et al. (2002). Appendix A provides detailed explanations of how to convert mass mixing ratios computed in the model into particle number densities, how these are used in computing size-averaged optical properties, and how to obtain radiometric properties of the atmosphere, such as aerosol optical depth (AOD) or backscattering coefficient $\beta_{\text {bak }}$, from the particles' optical properties and from the MATCH aerosol fields.

\subsubsection{Optics model for aerosol particles of different mixing states}

The new MATCH-optics model accounts for both internally and externally mixed aerosol particles, and it contains both homogeneously and inhomogeneously mixed aerosol particles. Different shapes and morphologies are assumed for different types of particles.

1. Pure, externally mixed black carbon particles are assumed to have a fractal aggregate morphology as shown in Fig. 1. The fractal morphology can be described by the statistical scaling law $N_{\mathrm{s}}=k_{0}\left(R_{\mathrm{g}} / a\right)^{D_{\mathrm{f}}}$, where $N_{\mathrm{S}}$ denotes the number of spherical monomers in the aggregate, $D_{\mathrm{f}}$ and $k_{0}$ are the fractal dimension and fractal prefactor, $a$ is the monomer radius, and $R_{\mathrm{g}}=$ $\sqrt{\sum_{n=1}^{N_{\mathrm{s}}} r_{n}^{2} / N_{\mathrm{s}}}$ is the radius of gyration, where $r_{n}$ describes the distance of the $n$th monomer from the aggregate's centre of mass. We use $D_{\mathrm{f}}=1.8, k_{0}=1.3$, which is based on the review in Bond and Bergstrom (2006). Although in the atmosphere black carbon aggregates may also have higher fractal dimensions (e.g. Adachi et al., 2007; Chakrabarty et al., 2014; China et al., 2014), assuming a fractal dimension around 1.8 yields 


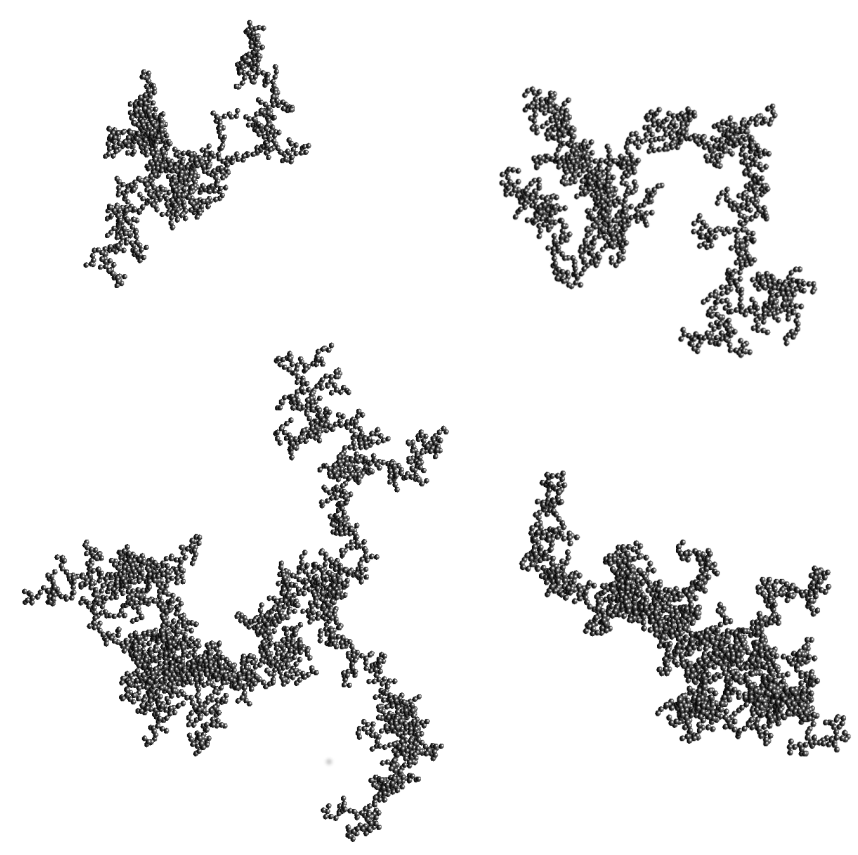

Figure 1. Examples of fractal aggregate model particles for computing optical properties of externally mixed black carbon. The aggregates consist of 1000, 1500, 2000, and 2744 monomers (in clockwise order, starting from upper left).

mass-absorption cross sections at $550 \mathrm{~nm}$ wavelength that lie closer to experimental data, as was shown in Kahnert and Devasthale (2011). The monomer radius can vary within a range of $10-25 \mathrm{~nm}$ (Bond and Bergstrom, 2006). Here it is assumed to be $a=25 \mathrm{~nm}$. This is consistent with field observations (Adachi and Buseck, 2008); also, it was shown (Kahnert, 2010b) that this choice of monomer radius in light scattering computations yields results for the single-scattering albedo of black carbon aggregates consistent with observations (Bond and Bergstrom, 2006).

The calculations in Kahnert and Devasthale (2011) were limited to aggregates up to $N_{\mathrm{s}}=600$. In order to cover the size range of externally mixed black carbon in SALSA we had to extend these calculations to aggregate sizes up to $N_{\mathrm{S}}=2744$, which corresponds to a volume-equivalent radius of $R_{\mathrm{V}}=350 \mathrm{~nm}$ (compare with Table 2). We used the multiple-sphere T-matrix code (Mackowski and Mishchenko, 2011), which is based on the numerically exact superposition T-matrix method for solving Maxwell's equations. Figure 2 shows some of the computed black carbon optical properties as a function of volume-equivalent particle radius and wavelength. All optical properties are averaged over particle orientations, where the orientationaveraging is performed analytically (Khlebstov, 1992). The absorption cross section $C_{\mathrm{abs}}$ shows the characteristic decline at long wavelengths, where the refractive in- dex of black carbon is changing only slowly (Chang and Charalampopoulos, 1990). Also, $C_{\text {abs }}$ increases with particle size. For small particle sizes this increase goes as $\sim R_{\mathrm{V}}^{3}$, which is typical for the Rayleigh scattering regime (Mishchenko et al., 2002).

2. Black carbon particles that are internally mixed with other aerosol components are morphologically very complex. It is technically beyond the reach of our present capabilities to build an aerosol-optics database with the use of morphologically realistic model particles. However, it is possible to employ realistic model particles in reference computations for some selected cases. This has recently been done in different studies (Kahnert et al., 2013; Scarnato et al., 2013, 2015). In the study by Kahnert et al. (2013), optical properties of encapsulated aggregate model particles, such as the one shown in Fig. 3 (left), were computed in the range of $100-500 \mathrm{~nm}$ (volume-equivalent radius), for different black carbon volume fractions, and for wavelengths from the UV-C to the mid-IR. The morphological parameters characterizing these model particles were based on field observations (Adachi and Buseck, 2008); the coating material was sulfate. The computations were performed with the discrete dipole approximation (Yurkin and Hoekstra, 2007).

In Kahnert et al. (2013) the computational results were compared to those obtained with simple model particles, such as externally mixed homogeneous spheres, internally mixed homogeneous spheres, and concentric core-shell particles with a carbon core and a sulfate shell. The analysis revealed which morphological properties of the encapsulated aggregate particles had the dominant impact on the optical properties. There are two important properties: (1) the amount of carbon mass that interacts with the electromagnetic field has a major impact on the absorption cross section $C_{\text {abs }}$. In a coreshell model as well as in a model based on externally mixed homogeneous spheres, all of the black carbon is concentrated in a single sphere. Owing to absorption of the electromagnetic field, radiation does not penetrate deeply into this sphere. Hence much of the carbon mass is shielded from interacting with the field, resulting in an underestimation of $C_{\text {abs }}$ compared to the encapsulated aggregates, in which a much larger fraction of the carbon mass can contribute to the absorption of electromagnetic energy. By contrast, in a homogeneous internal-mixture model the black carbon is distributed evenly throughout the sulfate host, which allows too much of the carbon mass to interact with the field. This results in an overestimation of $C_{\text {abs }}$. (2) Compared to a bare black carbon aggregate, a coated aggregate has a larger geometric cross section. Hence more light is intercepted by an internally mixed particle and focused on the black carbon inclusion, thus enhancing $C_{\mathrm{abs}}$. This 

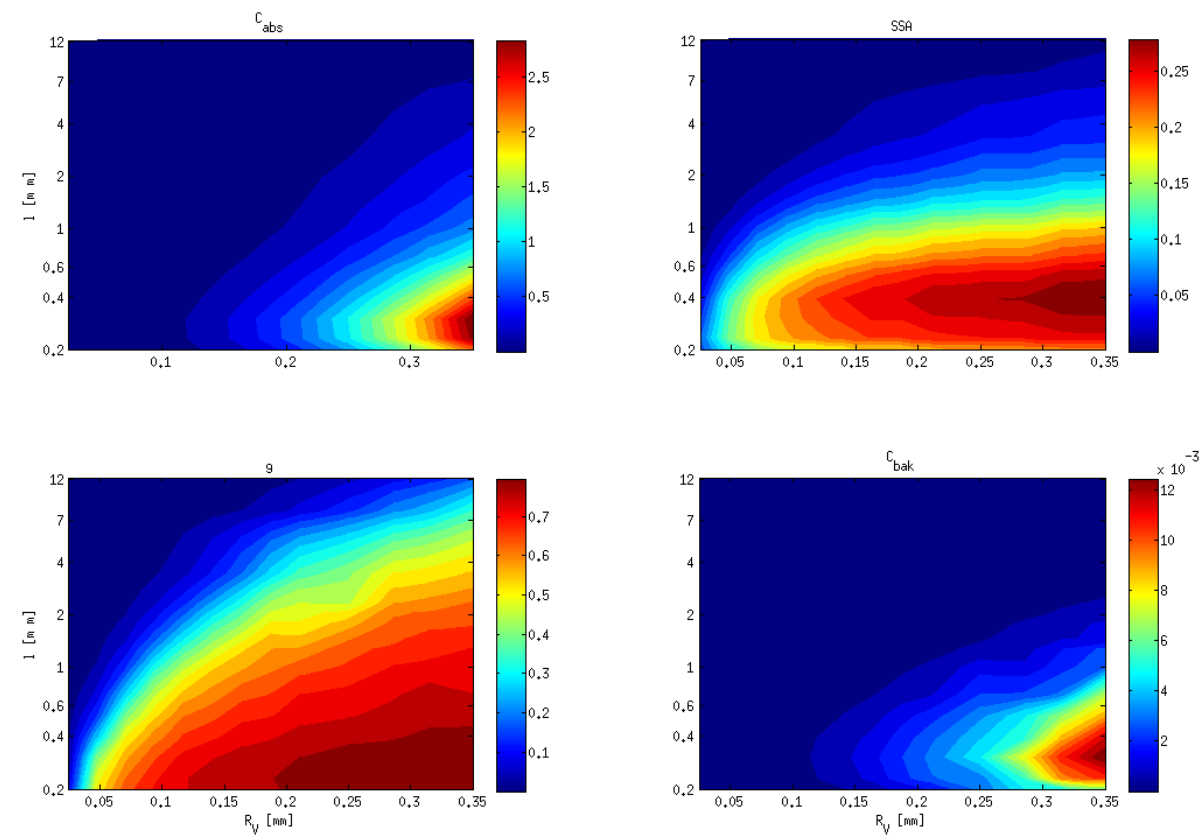

Figure 2. Absorption cross section $C_{\text {abs }}$ (upper left), single-scattering albedo SSA (upper right), asymmetry parameter $g$ (bottom left), and backscattering cross section $C_{\mathrm{bak}}$ (bottom right) of black carbon aggregates as a function of volume-equivalent radius $R_{\mathrm{V}}$ and wavelength $\lambda$.

effect is neglected in the external-mixture model, resulting in an underestimation of $C_{\text {abs }}$.

Note that in earlier studies (e.g. Jacobson, 2000) it was often tacitly assumed that a core-shell model would give the most accurate estimates of the aerosol optical properties, owing to its morphological similarity to encapsulated black carbon particles. However, the results in Kahnert et al. (2013) have clearly shown the shortcomings of the conventional core-shell model. But once we understand which morphological properties are most essential, and which ones make a minor contribution to the optical properties, we can devise model particles that account for the most important morphological effects, yet are sufficiently simple for computing a look-up table for large-scale modelling. It was proposed in Kahnert et al. (2013) to use a core-shell model (hence accounting for the coating effect) in which only part of the carbon mass is contained in the core, and the remaining part is homogeneously mixed with the shell. The model particle is illustrated in Fig. 3 (right). The fraction $f_{\mathrm{c}}$ of the carbon mass located in the core is a free parameter, with which one can interpolate between the two extreme models of the homogeneous mixture $\left(f_{\mathrm{c}}=0\right.$, all carbon mass mixed with the shell) and the regular core-shell model $\left(f_{\mathrm{c}}=1\right.$, all carbon mass in the core). This model has been referred to as the concentric core-grey-shell (CGS) model. The tuning of the free parameter $f_{\mathrm{c}}$ in the model was done to fit the reference model of encapsulated aggregates as described in Kahnert et al. (2013). It was found that $f_{\mathrm{c}}$ is independent of particle size, black carbon volume fraction, and of the optical property one wants to fit. However, $f_{\mathrm{c}}$ does depend on the wavelength of light.

The CGS model has been employed in generating the new MATCH-optics look-up table. The shell material can be any mixture of water-soluble components. We use the same values of $f_{\mathrm{c}}$ as those determined in Kahnert et al. (2013). Its dependence on wavelength is given in Table 3.

3. In the mass transport model, we assume that all SIA components and all sea salt are internally mixed. We furthermore assume that in size bins $1-4,0,70,70$, and $100 \%$, respectively, of the black carbon, $0,70,70$, and $70 \%$ of the organic carbon, and $0,1.3,1.3$, and $1.3 \%$ of the dust are internally mixed; the remaining BC, OC, and dust mass is externally mixed. In SALSA, the mixing state depends on the size bin (see Table 2), and the mixing proportions are provided by the model results. In both the mass transport model and in MATCH-SALSA, the contribution to the effective refractive index of dust and black carbon is computed by the Maxwell Garnett EMT (Maxwell Garnett, 1904), while for all other components we use the Bruggemann EMT (Bruggemann, 1935).

4. All other externally mixed particles not containing black carbon are assumed to be homogeneous spheres in the present version of the look-up table. 
Table 3. Core fraction $f_{\mathrm{c}}$ in the core-grey-shell model as a function of wavelength $\lambda$.

\begin{tabular}{lrrrrrrr}
\hline$\lambda(\mu \mathrm{m})$ & 0.2000 & 0.2316 & 0.3040 & 0.3400 & 0.3550 & 0.3800 & 0.3932 \\
$f_{\mathrm{c}}$ & 0.7 & 0.7 & 0.7 & 0.7 & 0.7 & 0.6 & 0.6 \\
\hline$\lambda(\mu \mathrm{m})$ & 0.4400 & 0.5000 & 0.5320 & 0.5332 & 0.6750 & 0.7016 & 0.8700 \\
$f_{\mathrm{c}}$ & 0.6 & 0.5 & 0.5 & 0.5 & 0.5 & 0.5 & 0.2 \\
\hline$\lambda(\mu \mathrm{m})$ & 1.0101 & 1.0200 & 1.064 & 1.2705 & 1.4625 & 1.7840 & 2.0460 \\
$f_{\mathrm{c}}$ & 0.1 & 0.1 & 0.1 & 0.1 & 0.1 & 0.1 & 0.1 \\
\hline$\lambda(\mu \mathrm{m})$ & 2.3250 & 2.7885 & 3.4615 & 3.5000 & 8.0205 & 10.600 & 12.195 \\
$f_{\mathrm{c}}$ & 0.1 & 0.1 & 0.1 & 0.1 & 0.1 & 0.1 & 0.1 \\
\hline
\end{tabular}
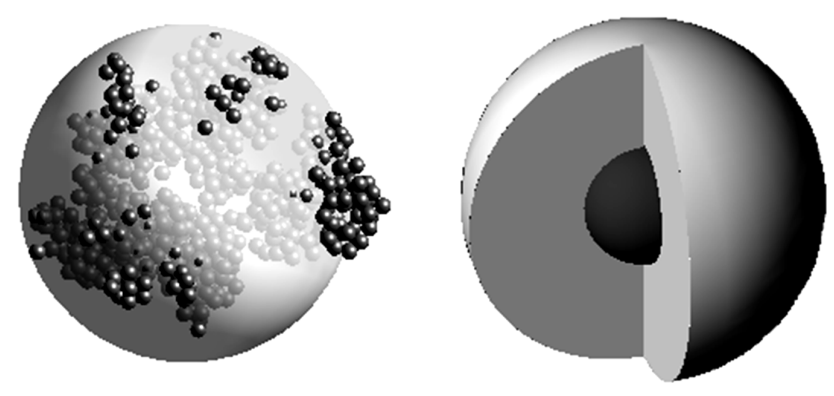

Figure 3. Morphologically realistic encapsulated aggregate model for internally mixed black carbon (left), and core-grey-shell model (right).

The look-up tables contain results for $C_{\text {ext }}, C_{\text {sca }}, g$, and $C_{\text {bak }}$ in 28 wavelength bands from the UV-C to the mid-IR. Computations with the CGS model were performed for 37 discrete $\mathrm{BC}$ volume fractions, namely, $f_{\mathrm{BC}}=0.00,0.01, \ldots$, $0.20,0.25, \ldots, 1.00$. For the shell material, as well as for non-carbon containing particles, the table contains (depending on the wavelength band) up to 40 discrete values of the real part and up to 18 discrete values of the imaginary part of the refractive index. The range of the refractive indices varies with wavelength; it is determined by those chemical components that, at each given wavelength, have the most extreme values of the refractive index. The optical properties are preaveraged over particle sizes for each size bin. Thus we generated one look-up table each for the mass transport model with its four size bins, and for SALSA with its 20 size bins. In SALSA it is assumed that the number density is constant in each size bin.

The MATCH-optics model computes in each grid cell and for each size bin the effective refractive index of the internally mixed material by use of EMT. The corresponding optical properties are obtained by linearly interpolating the closest pre-computed results in the look-up table. Size-averaging is performed by weighing the optical cross sections as well as $g \cdot C_{\text {sca }}$ in each size bin with the number density per bin and adding over all bins. The integrated quantities are then divided by the total particle number density; the integral over $g \cdot C_{\text {sca }}$ is also divided by the size-averaged scattering cross section.

\subsection{Methodology for comparing the optics models}

The new internal-mixture optics model with its BC fractal aggregate and core-grey-shell model particles accounts for significant morphological details in aerosol particles. The main question we want to address is whether or not this high level of detail is really necessary, i.e. whether it has any significant impact on optical properties modelled with a CTM. By significant we mean an impact that is comparable to other effects whose importance is well understood. Thus to make such an assessment we need to pick a well-understood effect that can serve as a gauge, i.e. to which we can compare the impact of particle morphology on optical properties. We take the effect of aerosol microphysics as a gauge. As aerosol microphysics is well known to have a substantial impact on aerosol concentrations and size distributions (Andersson et al., 2015; Matsui et al., 2013), this effect will provide us with a reference to which we can compare the impact of the morphological assumptions made in the aerosol-optics model. Thus we compute aerosol optical properties

1. with the MATCH mass-transport model (i.e. with aerosol microphysics switched off), in conjunction with the old optics model (abbreviated by MT-EXT, "masstransport external mixture");

2. with the MATCH mass-transport model in conjunction with the new optics model (MT-CGS, "mass-transport core-grey-shell");

3. with the MATCH-SALSA model (i.e. with aerosol microphysics switched on), in conjunction with the old optics model (abbreviated SALSA-EXT, "MATCHSALSA external mixture"); and

4. with the MATCH-SALSA model, in conjunction with the new optics model (SALSA-CGS, "MATCH-SALSA core-grey-shell").

We first perform a comparison of monthly and geographically averaged differences in aerosol optical properties. More 
specifically, comparison of model set-ups MT-EXT with MTCGS, or SALSA-EXT with SALSA-CGS allows us to assess the impact of the morphological assumptions in the optics model. Comparison of MT-EXT with SALSA-EXT, or MT-CGS with SALSA-CGS will give us an estimate of how much the inclusion or omission of microphysical processes impacts modelling results of aerosol radiometric properties.

While statistical analyses can uncover general trends, it is difficult to understand the underlying physical reasons for model differences from an analysis of temporally and geographically averaged model results. Therefore, we also consider a few case studies in some more detail. We take the optical properties modelled with different MATCH versions as input to a radiative transfer model and analyse the total aerosol radiative forcing and the black carbon radiative forcing. The main goal is to understand how differences in single-scattering optical properties between the two optics models impact the outcome of the radiative transfer simulations. To keep the case studies within manageable bounds, we restrict ourselves to four geographic locations (two over land, two over the ocean), two instances (one representing low-BC summer concentrations, one representing highBC winter conditions), and we limit ourselves to comparing model set-ups MT-EXT, MT-CGS, and SALSA-CGS. More specifically, we consider one site over northern Italy $\left(45.0^{\circ} \mathrm{N}, 8.5^{\circ} \mathrm{E}\right)$, one over the Mediterranean Sea $\left(37.5^{\circ} \mathrm{N}\right.$, $\left.5.5^{\circ} \mathrm{E}\right)$, one over Poland $\left(52.6^{\circ} \mathrm{N}, 21.0^{\circ} \mathrm{E}\right)$, and one over the North Sea $\left(52.0^{\circ} \mathrm{N}, 2.7^{\circ} \mathrm{E}\right)$. For the two instances, we pick 22 June 2007 12:00 UTC and 22 December 2007 12:00 UTC. Radiative transfer calculations are performed for each of these four sites and for both instances. Vertical profiles of the aerosol optical depth per layer, the single-scattering albedo, and the asymmetry parameter are used as input to the libRadtran radiative transfer package (Kylling et al., 1998), assuming a plane-parallel atmosphere. For the surface albedo of the ocean we assume a spectrally constant value of 0.065 , while for the spectrally varying surface albedo of the two land locations we used MODIS observations for each of the two instances. The results were spectrally integrated to obtain the broadband radiative fluxes. The radiative transfer simulations were repeated for corresponding profiles of optical properties (with a $1 \mathrm{~km}$ resolution) in the absence of black carbon, as well as for clear sky conditions. This allows us to compute differences in broadband radiative fluxes, i.e. the radiative effect of black carbon, and the radiative effect of all aerosol components. The results of this radiative transfer study are discussed in Sect. 3.2.

To further investigate the significance of the optics model for radiometric properties, we also look at optical properties relevant for remote sensing, namely, backscattering coefficient and Ångström exponent. These results are discussed in Sects. 3.3 and 3.4, respectively.

\section{Results}

\subsection{Aerosol optical properties in MATCH}

Figure 4 gives us a first impression of the differences between the four model configurations. The extinction AOD is shown for MT-EXT (first from the left), MT-GCS (second), SALSA-CGS (third), and SALSA-EXT (fourth). The overall spatial patterns are similar. This is expected, since all model configurations used the same EMEP emissions and HIRLAM meteorological data. However, the magnitude of the AOD results can differ significantly among the four model runs (note the semi-logarithmic scale!). It is also remarkable that the differences between the two optics models depend on whether we make this comparison within the mass-transport model (compare MT-EXT to MT-CGS), or within SALSA (compare SALSA-CGS and SALSA-EXT). It can also vary geographically. This merely confirms the complexity of aerosol-optics modelling. Replacing one optics model by another will not simply offset the resulting optical properties by some common factor; it will introduce a significant change in modelled optical properties, of which the magnitude and even the sign can be dependent on local conditions, such as the size distribution and the chemical composition of the aerosol particles.

This is also evident from a comparative analysis of geographically and temporally averaged aerosol optical properties. Table 4 shows aerosol optical depth (AOD), backscattering coefficient (BSCA), single scattering albedo (SSA), and asymmetry parameter $(g)$, each at three different wavelengths $(355,532$, and $1064 \mathrm{~nm})$, and each averaged over the whole model domain and over 1 month (December 2007). The columns show relative differences: for instance, MT $($ EXT,CGS $)=($ MT-EXT - MT-CGS $) /($ MTCGS) is the relative difference of MT-model results obtained with the EXT and CGS optics models.

Comparison of the columns "MT(EXT,CGS)" and "SALSA(EXT,CGS)" illustrates the differences between the optics models in the absence and presence of aerosolmicrophysical processes. As we already suspected from inspection of Fig. 4, differences in the optics models defy simplistic explanations; both the magnitude and sign of these difference can be strongly dependent on the size-dependent chemical composition and mixing state of the aerosols, hence on the model version with which the optics models are being compared. In our case, we see that compared to the CGS model, the EXT-optics model predicts higher values of AOD, BSCA, and $g$ in the MT model, and lower values of SSA. In SALSA the roles of the CGS and EXT model are reversed.

The "CGS(MT,SALSA)" column shows differences between optical properties computed in the absence and presence of aerosol-microphysical processes, where the optics computations have been performed with the CGS model. The "EXT(MT,SALSA)" column shows an analogous comparison, where the optics computations have 


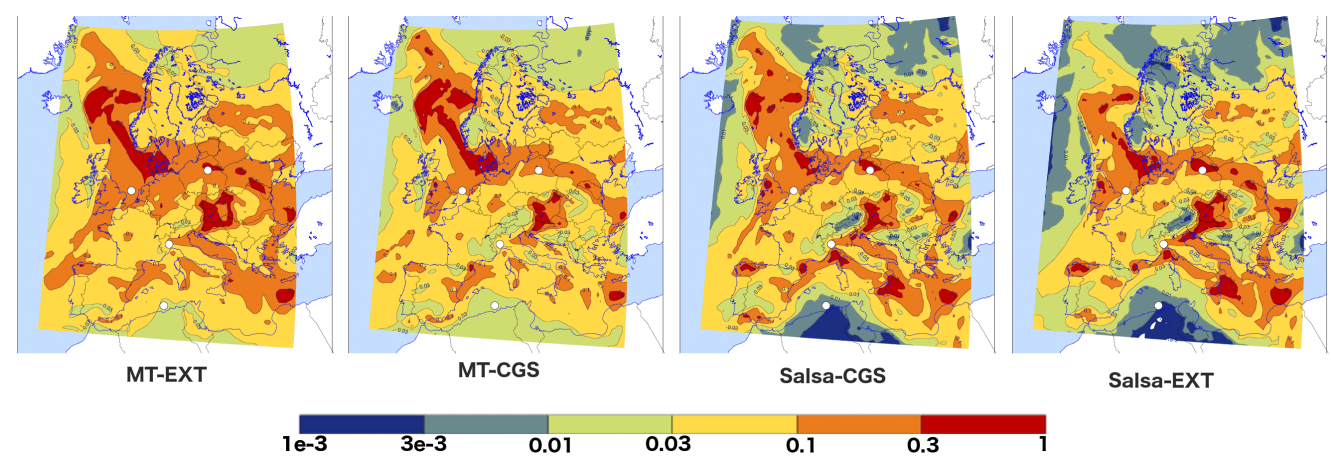

Figure 4. Aerosol optical depth over Europe on 22 December 2007, 12:00 UTC (noon). Results are shown for the mass transport model in conjunction with the old external-mixture optics model (first to the left), and with the new internal-mixture/core-grey-shell/fractal BC aggregate model (second to the left), as well as for the MATCH-SALSA model in conjunction with the new optics model (third to the left) and old optics model (fourth to the left). The circles indicate the four locations used for radiative transfer studies. Note the semi-logarithmic colour scale!

Table 4. Averaged relative difference in aerosol optical depth (AOD), backscattering coefficient (BSCA), single scattering albedo (SSA) and asymmetry parameter $(g)$, among the different model set-ups for December 2007. The average has been performed over a whole month and over all grid cells (horizontally for AOD, horizontally and vertically for all other optical properties). Each number corresponds to a relative difference between two model set-ups. For instance, MT(EXT,CGS) $=($ MT-EXT - MT-CGS)/(MT-CGS) compares results obtained with the mass transport model (MT) by using the two different optics models (EXT and CGS).

\begin{tabular}{lrrrr}
\hline$X_{\lambda}(\mathrm{nm})$ & MT(EXT,CGS) & SALSA(EXT,CGS) & CGS(MT,SALSA) & EXT(MT,SALSA) \\
\hline AOD $_{355}$ & 0.16 & -0.28 & -0.50 & -0.19 \\
AOD $_{532}$ & 0.08 & -0.14 & 0.00 & 0.25 \\
AOD $_{1064}$ & 0.18 & -0.03 & 0.14 & 0.37 \\
\hline BSCA $_{355}$ & 0.44 & -0.01 & -0.47 & -0.23 \\
BSCA $_{532}$ & 0.26 & -0.08 & -0.19 & 0.11 \\
BSCA $_{1064}$ & 0.99 & -0.01 & -0.36 & 0.28 \\
\hline SSA $_{355}$ & -0.02 & 0.04 & 0.03 & -0.03 \\
SSA $_{532}$ & -0.02 & 0.05 & 0.04 & -0.02 \\
SSA $_{1064}$ & -0.07 & 0.08 & 0.08 & -0.07 \\
\hline$g_{355}$ & 0.06 & -0.01 & -0.10 & -0.03 \\
$g_{532}$ & 0.10 & -0.00 & -0.06 & 0.04 \\
$g_{1064}$ & 0.17 & -0.02 & -0.11 & 0.06 \\
\hline
\end{tabular}

been performed with the EXT model. If we compare the magnitudes of the entries in columns "MT(EXT,CGS)" and "SALSA(EXT,CGS)" with the corresponding magnitudes of the entries in columns "CGS(MT,SALSA)" and "EXT(MT,SALSA)", then we see that the differences between the two optics models (EXT,CGS) are roughly of the same order as the differences between the two aerosol models (MT,SALSA). Thus, the main observation is that the choice of aerosol-optics model can have an effect on modelled optical properties that is of comparable magnitude to the level of detail in the description of aerosol-microphysical processes.

While spatio-temporally averaged model results allow us to draw some general conclusions, it is difficult to understand the reasons for the observed differences from such an analysis. We will, therefore, complement this investigation in the following sections with a more detailed analysis of some selected case studies.

\subsection{Optical properties and radiative forcing}

In Sect. 2.3.2 we have discussed how morphological properties of aerosol particles can impact their optical properties. We now take this one step further and discuss how the optical properties of particles can impact the radiative properties of an aerosol-laden atmosphere. We will show results for a single wavelength near the maximum of the solar spectrum. The comparison will focus on three model set-ups, MTEXT, MT-CGS, and SALSA-CGS. We will use MT-CGS as a reference and compare it first to SALSA-CGS in order to investigate the impact of aerosol-microphysics (MT versus SALSA). Next, we compare MT-CGS to MT-EXT in order 
Aerosol forcing, North Italy, 2007-06-22
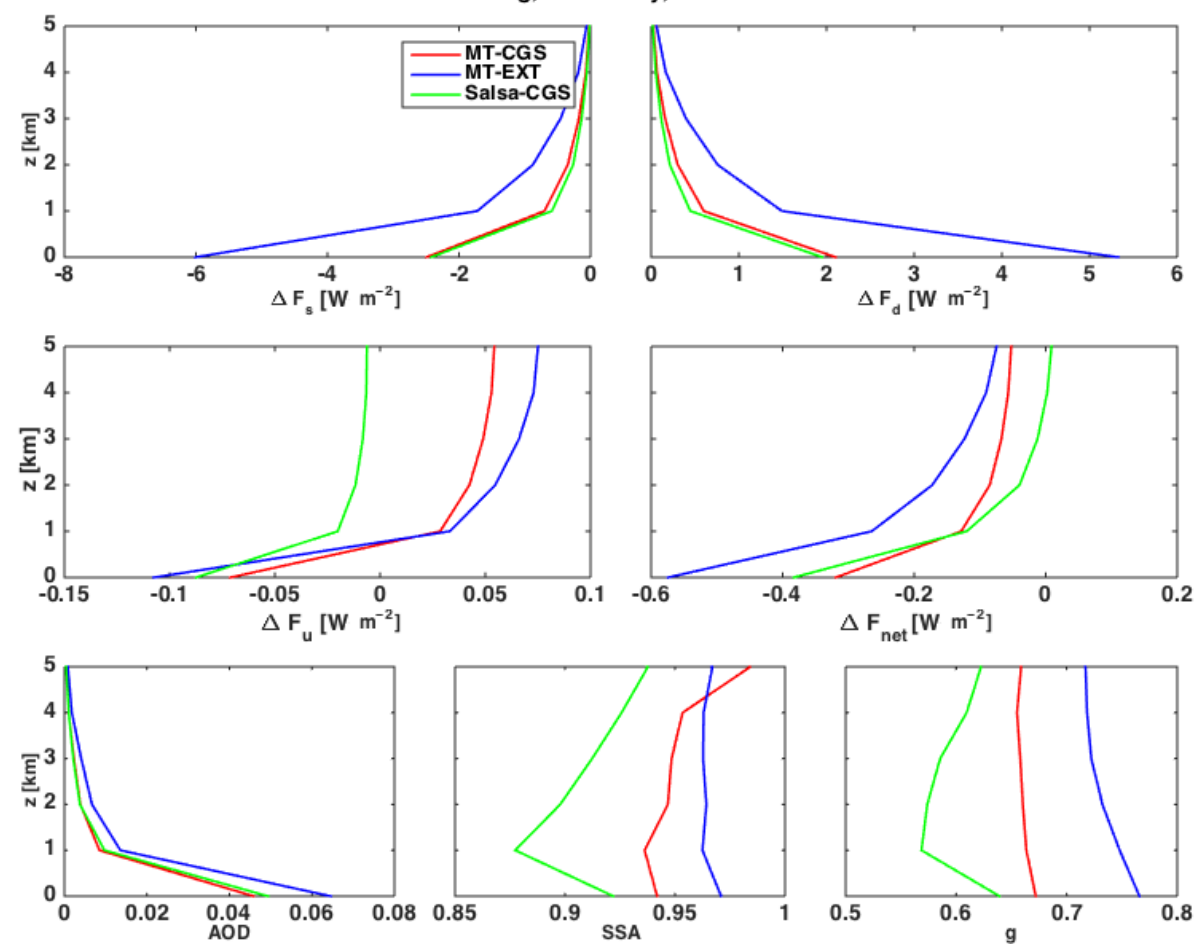

Figure 5. Aerosol forcing and optical properties at 532(CGS)/500(EXT) nm over northern Italy in June. Results are shown for the three model versions MT-EXT (blue), MT-CGS (red), and SALSA-CGS (green). The aerosol forcing is derived by taking the difference in radiative fluxes between an aerosol-laden and clear sky. The difference in direct $\left(\Delta F_{\mathrm{S}}\right)$ and diffuse $\left(\Delta F_{\mathrm{d}}\right)$ downwelling, as well as the diffuse upwelling $\left(\Delta F_{\mathrm{u}}\right)$ and the net radiative flux (aerosol forcing) $\left(\Delta F_{\text {net }}=\Delta F_{\mathrm{S}}+\Delta F_{\mathrm{d}}-\Delta F_{\mathrm{u}}\right)$, are shown in the first four figures (first and second row of plots). The optical properties aerosol optical depth (AOD), single scattering albedo (SSA), and asymmetry parameter $(g)$ are shown in the third row of plots.

to investigate the impact of the optics model (CGS versus EXT).

The result for the optical properties obtained with the three model versions (AOD per layer, SSA, and $g$ ) at the wavelength $532(\mathrm{CGS}) / 500(\mathrm{EXT}) \mathrm{nm}$, together with the radiative forcing for all aerosol components and black carbon, respectively, can be seen in Figs. 5-10 for northern Italy and the Mediterranean on 22 June 2007. Each figure shows the differences in direct solar flux $\Delta F_{\mathrm{s}}$ (top left), diffuse downwelling flux $\Delta F_{\mathrm{d}}$ (top right), diffuse upwelling flux $\Delta F_{\mathrm{u}}$ (centre left), and net radiative flux $\Delta F_{\text {net }}=\Delta F_{\mathrm{s}}+$ $\Delta F_{\mathrm{d}}-\Delta F_{\mathrm{u}}$ (centre right), where either the difference between aerosol-laden and clear sky conditions is considered (Figs. 5 and 6), or the difference between fluxes in the presence and absence of black carbon (Figs. 9 and 10). Here, downwelling fluxes are obtained by integrating the radiance over all azimuthal angles, and over polar angles from 90 to $180^{\circ}$, where the positive $z$ axis is directed from the ground to the top-of-atmosphere (TOA). Similarly, the upwelling flux is obtained by integrating the radiance over all azimuthal angles, and over polar angles from 0 to $90^{\circ}$. TOA results for the other geographical locations are summarized in Table 5 in terms of aerosol forcing $\left(\Delta F_{\text {net }}=\right.$
$F_{\text {net }}($ with aerosol particles $)-F_{\text {net }}$ (no aerosol particles)), and in Table 6 in terms of black carbon forcing $\left(\Delta F_{\text {net }}=\right.$ $F_{\text {net }}$ (with black carbon) $-F_{\text {net }}$ (no black carbon)). The wavelengths 532(CGS)/500(EXT) nm are near the maximum of the solar spectrum. Each figure has a vertical span of $5 \mathrm{~km}$, which comprises that part of the troposphere where most aerosol particles are concentrated in the cases we picked.

\subsubsection{Comparison of aerosol microphysics and mass-transport model}

We start by comparing radiative fluxes in the presence and absence of all aerosol components, which we refer to as the "aerosol radiative effects". Figures 5 and 6 show the aerosol radiative effects modelled over northern Italy and over the Mediterranean north of Algiers, respectively. The general patterns in both plots can be understood as follows. In the presence of all aerosol components, optical extinction is stronger than in clean air. Thus the presence of all aerosol components gives a reduction $\Delta F_{\mathrm{S}}$ in the direct solar flux (upper left). As the aerosol optical depth per atmospheric layer strongly increases near the ground, the magnitude of $\Delta F_{\mathrm{s}}$ increases sharply with decreasing altitude. Furthermore, 

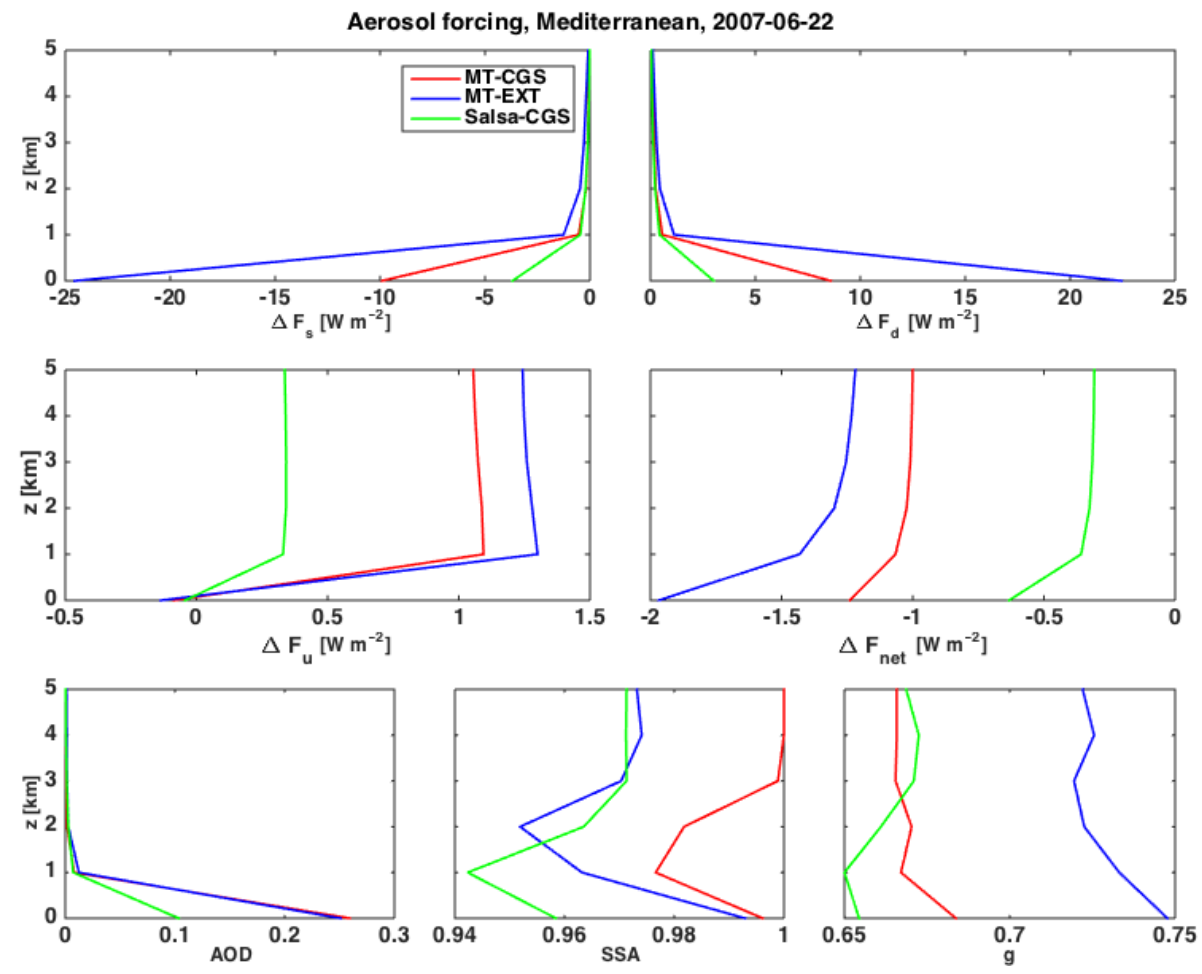

Figure 6. Same as Fig. 5 but over the Mediterranean.

extinction in the form of scattering results in the generation of diffuse flux; the downwelling diffuse flux accumulates downward, resulting in an increasing excess of downwelling flux $\Delta F_{\mathrm{d}}$ in the presence of aerosol components as one approaches the surface. The upwelling flux $F_{\mathrm{u}}$ is generated by scattering in the atmosphere and reflection at the surface. Since aerosol extinction reduces the net radiative flux as one approaches the surface, less upwelling diffuse flux is generated at low altitudes; hence, the difference in upwelling flux $\Delta F_{\mathrm{u}}$ between an aerosol-laden and a clear sky atmosphere is negative near the surface. However, it increases with altitude, because at higher altitudes the magnitude of the difference (aerosol-clear sky) in the net radiative flux that can be converted into upwelling diffuse flux decreases at higher altitudes.

If we focus now on differences in the radiative net flux $\Delta F_{\text {net }}$ at high altitudes, i.e. the radiative forcing effect of aerosol particles, then we see significant differences between the mass transport model (MT, red) and SALSA (green) at both geographic locations. It is evident that the main causes of these are corresponding differences in the diffuse upwelling flux $\Delta F_{\mathrm{u}}$.

At both locations the diffuse upwelling flux is smaller for SALSA then for MT, but for different reasons. Over the Mediterranean (Fig. 6), the AOD is significantly smaller for SALSA than for MT, resulting in less extinction of the direct flux, hence less generation of diffuse flux, and a smaller radiative cooling effect for SALSA.
Over northern Italy (Fig. 5), there is almost no difference in AOD between the two models. It can be seen from the AOD profile that the majority of aerosol particles reside in the lowest $1 \mathrm{~km}$ near the surface. However, above $1 \mathrm{~km}$ the results of $\Delta F_{\mathrm{u}}$ obtained with SALSA and MT diverge with increasing altitude. This is a result of the reflection by the nearsurface aerosol layer, which is different in the two models. In MT the SSA is higher than in SALSA, resulting in more scattering, and thus in more diffuse radiation. The asymmetry parameter is larger in MT than in SALSA; correspondingly, the partitioning in MT between downwelling and upwelling radiation is somewhat shifted in favour of the former. However, this only partially counteracts the generation of a higher amount of diffuse upwelling radiation in MT due to the higher SSA. The net effect is a higher value of $\Delta F_{\mathrm{u}}$ in MT, and hence a larger radiative cooling effect at higher altitudes.

To further analyse the difference in optical properties between MT and SALSA, we look at the aerosol masses and the relative sizes of the particles. Figure 7 shows vertical profiles of the effective radius $r_{\text {eff }}$ defined according to Eq. (1), in SALSA (green), and the MT model (black) over northern Italy (left) and over the Mediterranean (right).

$r_{\text {eff }}=\frac{\int_{0}^{\infty} n(r) \pi r^{2} \cdot r \mathrm{~d} r}{\int_{0}^{\infty} n(r) \cdot \pi r^{2} \mathrm{~d} r}$

Figure 8 shows profiles for the total aerosol mass (first row), BC (black carbon) (second row), sulfate (third row), and ni- 

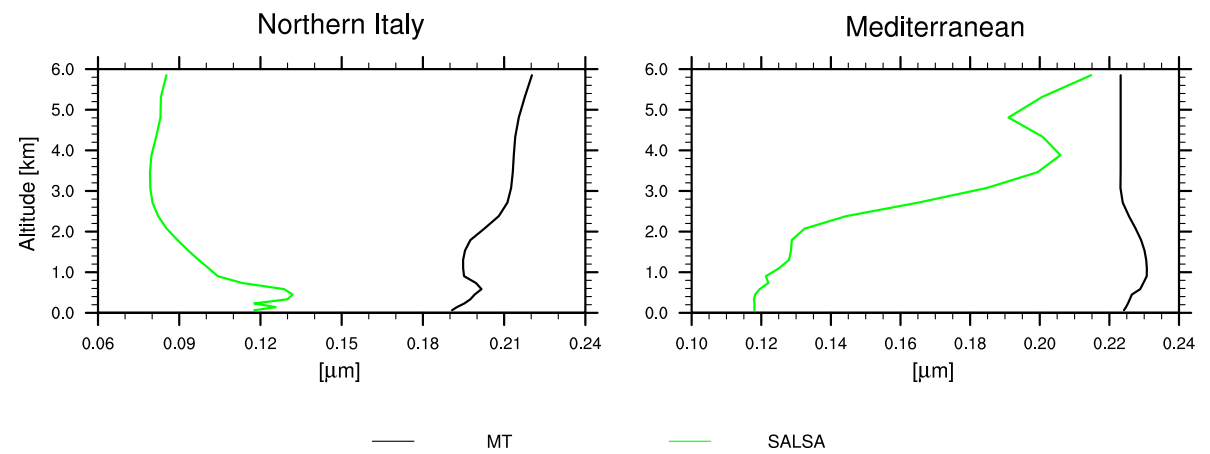

SALSA

Figure 7. Effective radius, $r_{\text {eff }}$, for the two chemical transport model versions MT and SALSA over northern Italy and the Mediterranean on 22 June 2007 at 12:00.

trate (fourth row) for both northern Italy (first column) and the Mediterranean (second column). We focus on the total aerosol mass, which is expected to impact the aerosol optical depth. The aerosol optical depth is dependent on the number density (which, in turn, increases with the mass mixing ratio), as well as on the extinction cross section (which generally increases with the effective radius of the particles). Over northern Italy, the SALSA model predicts a larger mass mixing ratio than the MT model (Fig. 8, upper left) and a smaller particle size (Fig. 7, left). This results in a higher number density but a smaller extinction cross section. These two effects cancel almost exactly, resulting in nearly identical aerosol optical depths predicted with the two models (Fig. 5, bottom left). By contrast, over the Mediterranean, the two models predict similar mass mixing ratios (Fig. 8, upper right), while SALSA predicts a much lower effective radius than the MT model (Fig. 7, right). As a consequence, the optical depth is significantly lower in SALSA than in MT (Fig. 6, bottom left). The SSA is lower in SALSA than in MT. This is mainly caused by the fact that the effective radius is smaller in SALSA than in MT, since SSA is usually increasing with size.

For the other two geographical locations and the second time event, the TOA results are summarized in Table 5. Over the North Sea, northern Italy, and the Mediterranean, the TOA forcing is strongest in the MT-EXT model (mass transport with old optics model), it is smaller in the MT-CGS model (mass transport with new optics model), and weakest in the SALSA-CGS model (aerosol-microphysics with new optics model). However, over Poland the negative forcing in SALSA-CGS is strongest among the three models in the summer, and second strongest in the winter. This can be explained by SALSA having a larger amount of aerosol particles throughout the column at that site, especially more sulfate, which, when externally mixed, contributes to a larger amount of scattering and therefore a higher SSA and a larger diffuse upwelling radiative flux.

We now compare radiative fluxes in the presence and absence of black carbon, which we refer to as the "black carbon radiative effect". Figures 9 and 10 show the radiative effect of black carbon together with the optical properties with and without black carbon. Again, differences in $\Delta F_{\text {net }}$ at TOA are mainly caused by corresponding differences in the upwelling diffuse radiative flux $\Delta F_{\mathrm{u}}$. In these figures, we have to focus on the difference in the optical properties when analysing the radiative fluxes. The general pattern can be seen in Fig. 9, which shows the differences in radiative fluxes and in the optical properties over northern Italy. The direct solar flux (upper left) decreases with decreasing altitude owing to extinction. The magnitude of the decrease mainly reflects the differences in optical depth in the presence and absence of black carbon (bottom left), which is larger in SALSA than in MT. The decrease in solar flux does not automatically result in an increase in the downwelling diffuse flux with decreasing altitude (upper right), as it was in the comparison of fluxes in the presence and absence of all aerosol components. The situation is more complex now. Near the surface, where the optical depth is largest, the difference in SSA in the presence and absence of black carbon is large in the MT model (bottom centre, red lines), and smaller in SALSA (green lines). As a result, absorption contributes more to the total extinction in the MT model than in SALSA (at least near the surface). Hence, the portion of the downwelling flux that is absorbed on its way downward is larger in the MT model than in SALSA, resulting in a decrease of the diffuse downwelling flux with decreasing altitude (upper right, red line). The differences between the two models in the diffuse upwelling flux are very small (centre left, red and green lines). This is the result of cancelling effects; for instance, there is less direct solar flux, but more diffuse downwelling flux in SALSA that can be converted into diffuse upwelling flux through scattering. As a result, the differences between both models in the net flux (centre right, red and green lines) are almost negligible.

Figure 10 shows the radiative effect of black carbon over the Mediterranean. Again, differences in $\Delta F_{\text {net }}$ at TOA are mainly caused by corresponding differences in the upwelling diffuse radiative flux $\Delta F_{\mathrm{u}}$. MT and SALSA only differ by a few $\mathrm{mW} \mathrm{m}^{-2}$. The main reason is that the optical properties, 

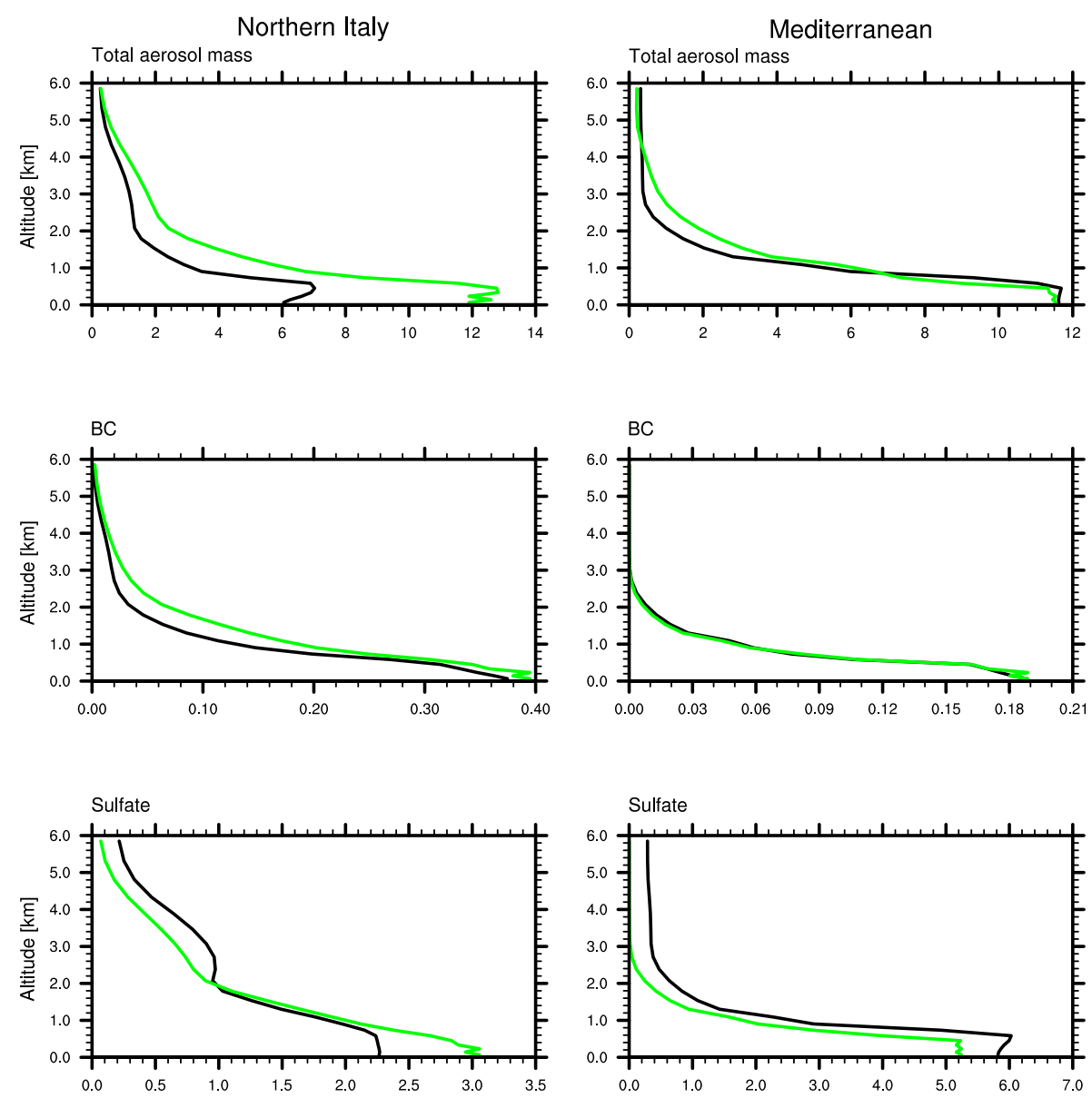

MT

SALSA

Figure 8. Vertical distribution of aerosol particles in northern Italy and over the Mediterranean on 22 June 2007 at 12:00.

especially the SSA, differ in the presence and absence of BC by almost the same amount in both models.

Table 6 shows the black carbon forcing for all the four geographical locations and both months. As a general trend, large differences in $\mathrm{BC}$ concentrations are visible as corresponding differences in $\mathrm{BC}$ forcing. For instance, nearsurface $\mathrm{BC}$ winter concentrations in northern Italy are about a factor of 10 higher than in summer; summer concentrations over the Mediterranean are more than a factor of 2 higher than in winter; in northern Italy in winter, SALSA predicts more than 2 times higher $\mathrm{BC}$ concentrations than the mass-transport model, while over the Mediterranean in winter the role of the two models is reversed (not shown). All of this corresponds to the respective $\mathrm{BC}$-forcing rates in Table 6. However, when the differences in $\mathrm{BC}$ concentrations are small, then there is no clear relation between the concentration differences and the corresponding differences in BC-forcing rates. For instance, as we see in Fig. 8, there is almost no difference between the $\mathrm{BC}$ concentrations com- puted with the two models over the Mediterranean in summer. But the table shows that the mass-transport model predicts a slightly higher forcing rate than SALSA. A possible cause is the difference in the size distributions in SALSA and the mass-transport model.

\subsubsection{Comparison of the two optics models}

The comparison between SALSA and the MT model in the previous section served two purposes. First, it helped us to develop a basic understanding of the effects of aerosol particles and black carbon on radiative fluxes. Second, it provided us with a gauge for assessing the importance of the aerosoloptics model, which will be the subject of this section.

We compare the old EXT (blue line) and the new CGS (red line) optics models in Figs. 5 and 6, each used in conjunction with the MT version of MATCH. The net radiative flux $\Delta F_{\text {net }}$ in the CGS model shows a weaker TOA cooling effect than the EXT model, both over northern Italy and over the 
Table 5. The aerosol forcing at the top of the atmosphere (TOA), $\Delta F_{\text {net,TOA }}\left(\mathrm{W} \mathrm{m}^{-2}\right)$, for the four different geographical locations, one summer (22 June 2007, 12:00) and one winter (22 December 2007, 12:00) event, and three model versions, MT-EXT, MTCGS, and SALSA-CGS.

\begin{tabular}{llrrr}
\hline \multirow{6}{*}{ Summer } & & MT-EXT & MT-CGS & SALSA-CGS \\
& Poland & -0.21 & -0.21 & -0.77 \\
& North Sea & -0.34 & -0.29 & -0.24 \\
& Northern Italy & -0.06 & -0.05 & 0.01 \\
& Mediterranean & -1.20 & -0.99 & -0.30 \\
\hline \multirow{2}{*}{ Winter } & Poland & -4.41 & -2.00 & -2.18 \\
& North Sea & -2.85 & -1.21 & -0.86 \\
& Northern Italy & -1.15 & -0.53 & -0.57 \\
& Mediterranean & -0.09 & -0.04 & -0.03 \\
\hline
\end{tabular}

Mediterranean. Again, the upwelling flux has the dominant impact on the behaviour of the TOA net radiative flux. Over northern Italy (Fig. 5) the diffuse upwelling flux is larger for the EXT model above $1 \mathrm{~km}$, whereas it is smaller below $1 \mathrm{~km}$ and at the bottom of the atmosphere (BOA). The AOD profile reveals that in the EXT model extinction is stronger than in the CGS model throughout the tropospheric column. As a result, there is more diffuse downwelling flux being generated in EXT than in CGS. At the bottom of the atmosphere (BOA) extinction of the direct flux is stronger than generation of diffuse downwelling flux; hence, less downwelling flux is reflected by the surface, resulting in less BOA upwelling diffuse flux in EXT than in CGS. Higher up in the troposphere, the upwelling diffuse flux is mostly generated by atmospheric scattering rather than reflection from the surface. As the SSA in EXT is higher than in CGS, more diffuse flux is generated, resulting in a stronger radiative cooling effect in EXT than in CGS.

Over the Mediterranean (Fig. 6), the EXT and CGS models have almost identical AOD profiles in the green part of the spectrum. However, at longer wavelengths EXT predicts substantially higher AOD values than CGS (see the Supplement provided with figures of the different optical properties and wavelengths). For instance, at $\lambda=1020 \mathrm{~nm}$ the near-surface AOD per layer computed with the EXT model is about twice as high as that computed with the CGS model. This explains the larger amount of diffuse broadband radiation generated in the EXT model, which results in a stronger negative TOA net flux in EXT as compared to the CGS model. Note that the differences in SSA between EXT and CGS are less than 0.03 , while the differences in $g$ are as much as 0.07 . The higher values of $g$ in EXT may contribute to the large amount of diffuse downwelling radiation in that model; however, the dominant effect is likely to be the high optical depth at red and IR wavelengths (Supplement).

Table 5 summarizes the TOA net radiative flux at all four geographical locations for both June and December. The largest differences among the models are seen in Decem-
Table 6. Same as Table 5 but for black carbon forcing.

\begin{tabular}{llrrr}
\hline & & MT-EXT & MT-CGS & SALSA-CGS \\
\hline \multirow{2}{*}{ Summer } & Poland & $1.02 \times 10^{-2}$ & $1.16 \times 10^{-2}$ & $1.20 \times 10^{-2}$ \\
& North Sea & $1.71 \times 10^{-2}$ & $1.54 \times 10^{-2}$ & $1.49 \times 10^{-2}$ \\
& Northern Italy & $4.61 \times 10^{-2}$ & $7.77 \times 10^{-2}$ & $7.86 \times 10^{-2}$ \\
& Mediterranean & $8.54 \times 10^{-3}$ & $6.45 \times 10^{-3}$ & $2.41 \times 10^{-3}$ \\
\hline \multirow{2}{*}{ Winter } & Poland & $4.03 \times 10^{-2}$ & $3.56 \times 10^{-2}$ & $6.83 \times 10^{-2}$ \\
& North Sea & $1.95 \times 10^{-2}$ & $2.28 \times 10^{-2}$ & $4.97 \times 10^{-3}$ \\
& Northern Italy & $6.73 \times 10^{-2}$ & $1.08 \times 10^{-1}$ & $1.46 \times 10^{-1}$ \\
& Mediterranean & $6.03 \times 10^{-4}$ & $1.34 \times 10^{-3}$ & $3.13 \times 10^{-4}$ \\
\hline
\end{tabular}

ber at the two northernmost locations, i.e. Poland and the North Sea. At these two places, the total aerosol amount (not shown) is significantly higher than at the other two locations farther south, giving rise to larger absolute changes in the aerosol forcing.

The black carbon forcing in Figs. 9 (northern Italy) and 10 (Mediterranean) display different behaviours in radiative fluxes, comparing the EXT (blue) and CGS (red) model results. Over northern Italy, the net black carbon forcing is more significant than over the Mediterranean in Fig. 10 due to higher levels of BC; see Fig. 8. As can be seen in Fig. 9, the differences in optical properties computed with and without black carbon are larger in the CGS model than in the EXT model, particularly for the SSA. This means that in the CGS model the presence of black carbon causes more absorption than in the EXT model, thus generating less diffuse downwelling and upwelling flux by scattering. As a result, the CGS model predicts more radiative warming, i.e. a higher TOA radiative net flux than the EXT model. The reason for this is that (i) the CGS model treats externally mixed soot as aggregates, which have a lower SSA than the massive black carbon spheres in the EXT model; and (ii) the CGS model treats internally mixed soot as a coated core-grey-shell model, which accounts for focusing of electromagnetic radiation onto the carbon core, thus enhancing absorption, i.e. lowering the SSA, while the EXT model treats all black carbon as externally mixed.

Let us now return to the main question of this study, namely, whether or not the level of detail in aerosol optics modelling has a significant impact on observable radiometric properties. We already saw in Table 4 that, on average, the effect of including aerosol microphysics on optical properties is of comparable magnitude as the effect caused by the morphological assumptions in the aerosol optics model. However, we also saw that the magnitude and sign of these impacts can be quite variable and depend on several factors. We find this confirmed in the analysis of our radiative-transfer study. More specifically, we can compare in Figs. 5-10 the differences in radiative forcing between MT-CGS and SALSA-CGS (red and green lines) to the corresponding differences between MT-CGS and MT-EXT (red and blue lines). We see that in some cases the choice of op- 

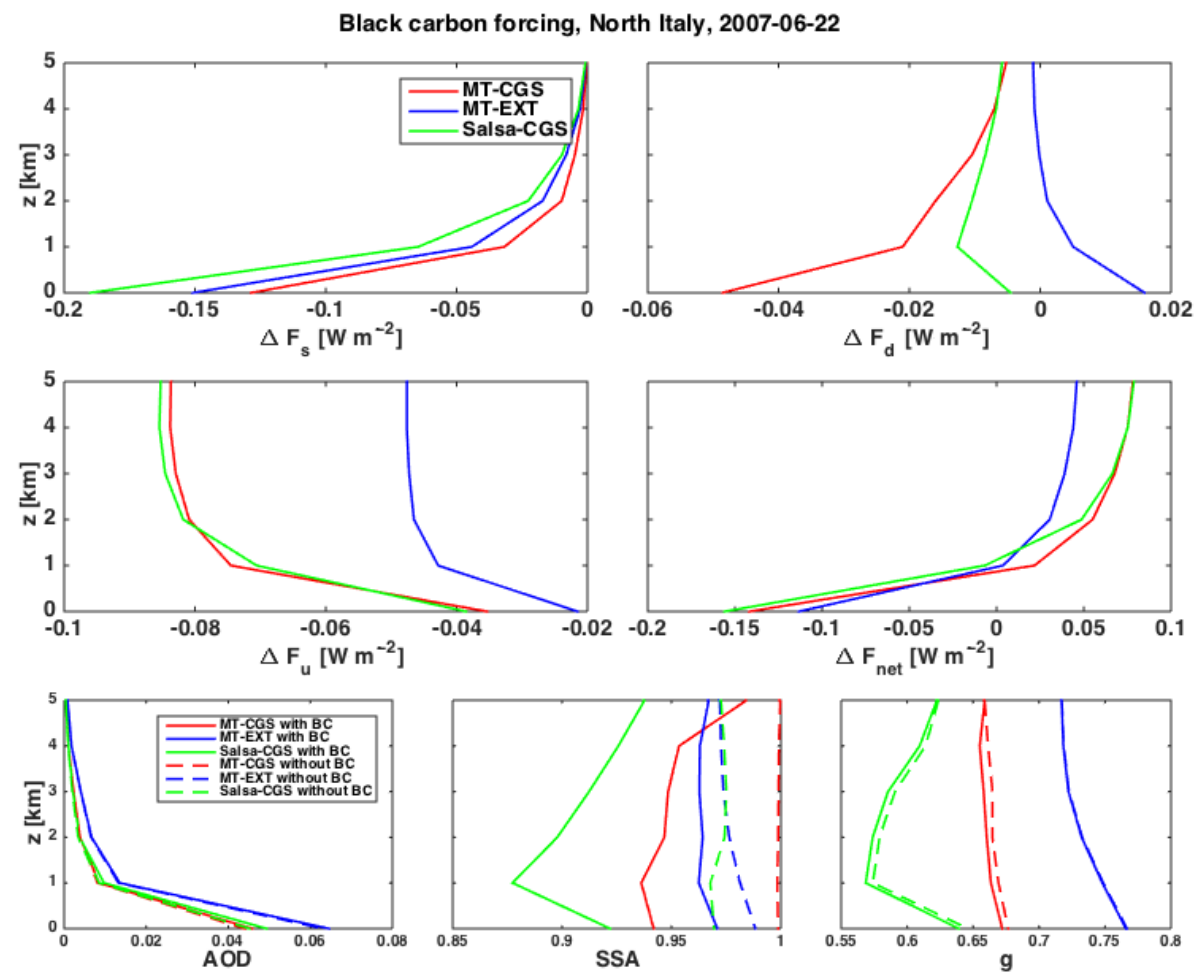

Figure 9. Black carbon forcing and optical properties at 532(CGS)/500(EXT) nm over northern Italy in June. Results are shown for the three model versions MT-EXT (blue), MT-CGS (red), and SALSA-CGS (green). The black carbon forcing is derived by taking the difference in radiative fluxes between an aerosol-laden sky including black carbon and an aerosol-laden sky omitting black carbon. The difference in direct $\left(\Delta F_{\mathrm{S}}\right)$ and diffuse $\left(\Delta F_{\mathrm{d}}\right)$ downwelling, as well as the diffuse upwelling $\left(\Delta F_{\mathrm{u}}\right)$ and the net radiative flux (aerosol forcing) $\left(\Delta F_{\text {net }}=\right.$ $\Delta F_{\mathrm{s}}+\Delta F_{\mathrm{d}}-\Delta F_{\mathrm{u}}$ ), are shown in the first four figures (first and second row of plots). The optical properties aerosol optical depth (AOD), single scattering albedo (SSA), and asymmetry parameter (g) are shown in the third row of plots.

tics model has a stronger effect than the inclusion of aerosol microphysics (e.g. Fig. 9), while in other cases it is the other way round (e.g. Fig. 6). We can also inspect Tables 5 and 6 and arrive at the same result.

In the following two subsections, we will focus on the selected case studies and discuss the significance of the optics model for radiometric quantities that are relevant for remote sensing applications.

\subsection{Backscattering coefficient}

From ground-based and space-borne lidar measurements one can obtain the aerosol backscattering coefficient $\beta$, which is proportional to the backscattering cross section $C_{\text {bak }}$ of the particles and the aerosol number density. Figure 11 shows vertical profiles of $\beta$ computed at two locations and at two instances, as indicated in the figure headings. Each panel shows computational results obtained with the three different model versions. The figure shows results for the second Nd:YAG harmonic of $532 \mathrm{~nm}$. Corresponding results computed for wavelengths of 355 and $1064 \mathrm{~nm}$ lead to similar conclusions.
We saw in Fig. 8 for June over northern Italy (upper left) that SALSA predicts an aerosol mass mixing ratio, and hence a particle number density that is higher than that in the MT model. But we also saw in Fig. 7 (left) that SALSA predicts lower values of $r_{\text {eff }}$. This results in lower values of $C_{\text {bak }}$. We see in Fig. 11 (upper left) that the effect on $\beta$ of the higher number density dominates over the effect of the lower $r_{\text {eff }}$, resulting in values of $\beta$ that are about $30 \%$ higher in SALSA (green line) than in the MT model (red line). Over the Mediterranean, both SALSA and the MT model predict similar mass mixing ratios (Fig. 8, upper right), but SALSA still predicts substantially lower values of $r_{\text {eff }}$ (Fig. 7, right). The result is that $\beta$ computed with the MT model (red line) is almost twice as high as the corresponding results obtained with SALSA (green line) (Fig. 11, upper right).

A similar comparison of the two optics models (red and blue lines in Fig. 11) shows that the new CGS optics model consistently predicts substantially lower values of $\beta$ than the old EXT optics model. This agrees with the comparison shown in Kahnert et al. (2013) between encapsulated black carbon aggregates and externally mixed homogeneous spheres. (In a retrieval algorithm, an optics model that overestimates the backscattering cross section would result in un- 
Black carbon forcing, Mediterranean, 2007-06-22
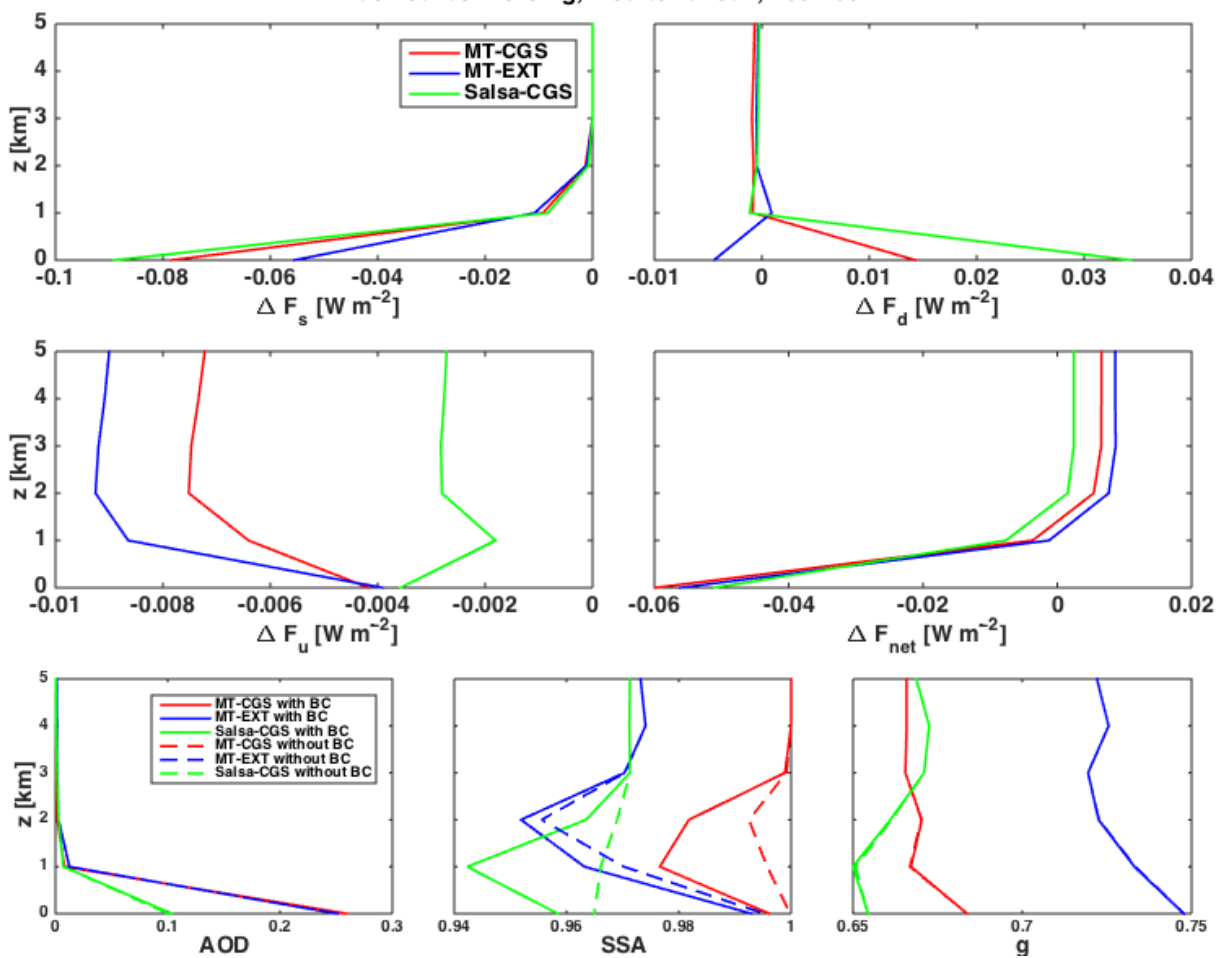

Figure 10. Same as Fig. 9 but over the Mediterranean.
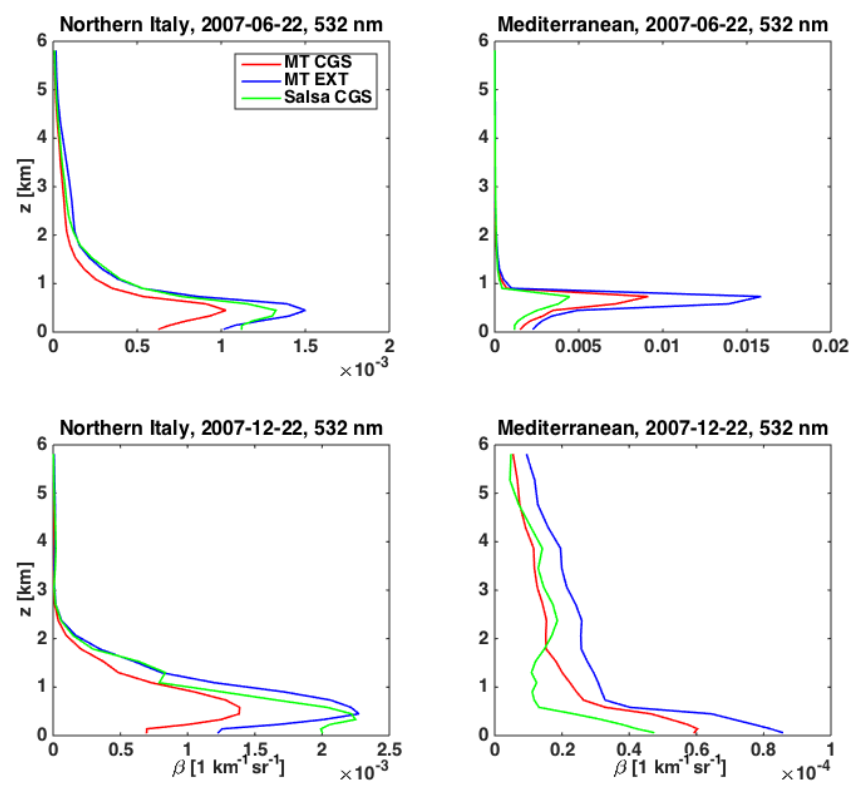

Figure 11. Backscattering coefficient at a wavelength of $532 \mathrm{~nm}$ in northern Italy and the Mediterranean and at two different time events (22/6 and 22/12/2007 at 12.00), computed with the three model versions: MT-EXT (blue), MT-CGS (red), and SALSA-CGS (green). derestimated retrieval results for the particle number density.) The differences between the two optics models are on the same order of magnitude (and often even larger) than the corresponding differences between the SALSA and the MT versions of the aerosol transport model.

\section{4 Ångström exponent}

The Ångström exponent $\alpha$ in a wavelength interval $\left[\lambda_{1}, \lambda_{2}\right]$ is defined as

$\alpha=-\frac{\log \left(\tau\left(\lambda_{1}\right) / \tau\left(\lambda_{2}\right)\right)}{\log \left(\lambda_{1} / \lambda_{2}\right)}$,

where $\tau$ denotes the extinction optical depth. This quantity is often used for obtaining particle size information (usually, the smaller the particle size, the larger $\alpha$ ). Table 7 shows values of $\alpha$ for our different test cases computed with the three model versions in the wavelength interval $532-1064 \mathrm{~nm}$. If we compare the columns labelled MT-CGS and SALSACGS, then we see that the mass-transport model consistently gives lower values of $\alpha$. This is related to the high values of $r_{\text {eff }}$ in that model, which we noted earlier. On the other hand, if we compare the columns labelled MT-EXT and MT-CGS, then we see that the new optics model (CGS) predicts higher values of $\alpha$ than the old model (EXT) in June (summer) for all four geographical locations and in December (winter) for locations Poland and the North Sea. This indicates that the errors introduced by the simple external-mixture model in 
Table 7. Angström exponent in the wavelength region 532$1064 \mathrm{~nm}$ for the four different geographical locations, one summer (22 June 2007, 12:00) and one winter (22 December 2007, 12:00) event, and three model versions, MT-EXT, MT-CGS, and SALSACGS.

\begin{tabular}{llrrr}
\hline & & MT-EXT & MT-CGS & SALSA-CGS \\
\hline \multirow{2}{*}{ Summer } & Poland & $0.32 \times 10^{0}$ & $0.12 \times 10^{1}$ & $0.28 \times 10^{1}$ \\
& North Sea & $0.80 \times 10^{0}$ & $0.12 \times 10^{1}$ & $0.21 \times 10^{1}$ \\
& Northern Italy & $0.11 \times 10^{1}$ & $0.11 \times 10^{1}$ & $0.15 \times 10^{1}$ \\
& Mediterranean & $0.36 \times 10^{0}$ & $0.12 \times 10^{1}$ & $0.21 \times 10^{1}$ \\
\hline \multirow{2}{*}{ Winter } & Poland & $0.80 \times 10^{0}$ & $0.12 \times 10^{1}$ & $0.22 \times 10^{1}$ \\
& North Sea & $0.79 \times 10^{0}$ & $0.11 \times 10^{1}$ & $0.14 \times 10^{1}$ \\
& Northern Italy & $0.13 \times 10^{1}$ & $0.10 \times 10^{1}$ & $0.12 \times 10^{1}$ \\
& Mediterranean & $0.13 \times 10^{1}$ & $0.98 \times 10^{0}$ & $0.14 \times 10^{1}$ \\
\hline
\end{tabular}

computing $\alpha$ are unpredictable, even the sign of the error. When used in a size retrieval algorithm the retrieval errors caused by the EXT model would be equally hard to predict. The difference between the MT and SALSA model is larger, but not much larger, than the differences between the old and new optics models. Note that the performance of the MT model could be improved in comparison to SALSA by modifying the assumed size distribution in the MT model. By contrast, the differences between the two optics models are rather fundamental; they are caused by the simple treatment of aerosol morphology in the EXT model.

\section{Conclusions}

We have implemented a new aerosol-optics model in a regional chemical transport model. The new model differs from an earlier optics model described in Kahnert (2008) in three essential points. (i) While the old model treats all chemical components as externally mixed, the new model accommodates both external and internal mixtures of aerosol species. (ii) The old model treats black carbon particles as homogeneous spheres; the new model assumes a fractal aggregate morphology with fractal parameters based on observations. Mass absorption cross sections and single scattering albedos computed with this model have previously been evaluated by comparison with measurements (Kahnert, 2010b). (iii) The new model describes internally mixed black carbon particles by a recently developed "core-grey-shell" model (Kahnert et al., 2013). This model accounts for the inhomogeneous internal mixing state of black carbon aggregates encapsulated in a shell of liquid-phase material. The model has been evaluated by comparison with reference computations based on observation-derived realistic models for encapsulated fractal aggregates (Kahnert et al., 2013). Item (i) has been incorporated in other CTMs earlier (e.g. Saide et al., 2013); however, to the best of our knowledge, items (ii) and (iii) go significantly beyond the current state-of-the-art of aerosol-optics models employed in CTMs. The main question of the present study is whether or not such a substantial level of detail in the description of aerosol morphology and optical properties is needed in a CTM.

We first performed a comparison of optical properties averaged over the entire model domain and over 1 month. To gauge the differences between the new and old optics models, we furthermore compare two model versions of the CTM with different levels of detail in the aerosol process descriptions, namely, one version that includes aerosoldynamic processes, and a simpler mass-transport model, in which aerosol microphysics is switched off. The importance of aerosol microphysics is well understood and can therefore serve as a reference. We found that the differences in optical properties between the two optics models are of the same order as those between the versions that include and exclude microphysical processes. For example, the aerosol optical depth computed with the two optics models differs by -28 to $18 \%$; differences obtained by inclusion or omission of aerosol microphysics are between -50 and $37 \%$. Corresponding differences in the backscattering coefficient are -8 to $99 \%$ and -47 to $28 \%$, respectively. Analogous observations can be made for other radiometric properties.

We furthermore wanted to understand how the differences in optical properties impact radiative transfer processes in an aerosol-laden atmosphere. To this end we compare radiative fluxes modelled with the old and new optics models. The comparison showed that the differences in radiative net fluxes between the two optics models are of similar magnitude as corresponding differences between the aerosolmicrophysics and mass-transport models.

These results strongly suggest that simplifications in the assumptions on aerosol morphology in the optics model can introduce substantial errors in modelled radiative fluxes and observables relevant to remote sensing. In chemistry-climate models such errors would enter into the simulation of the direct aerosol radiative forcing effect and add to all other sources of error in the model. In model evaluations that make use of remote sensing observations these errors would complicate the comparison between model results and observations.

The modifications to the morphology assumptions in the optics model were limited to black carbon particles. There are many other aerosol particles with complex morphological properties, such as mineral dust, which our optics model still treats by a simple homogeneous-sphere model. The findings of our study should be an incentive for improving the description of dust and volcanic-ash optical modelling in CTMs. A recent review of our current state of knowledge on aerosol morphology and aerosol optics for a variety of different aerosol particles can be found in Kahnert et al. (2014).

The findings of this study are likely to have implications for chemical data assimilation. In data assimilation one employs an observation operator that maps the model results to observable quantities. In the case of satellite-based observations of aerosol-optical properties, the observation operator is 
just our aerosol-optics model, possibly coupled to a radiative transfer model. Many data-assimilation methodologies, such as the variational method, require a linear (or, at least, linearized) observation operator. In the old optics model, which assumes externally mixed aerosol particles, the observation operator is, indeed, linear (Kahnert, 2008). This largely explains why external-mixture optics models are widely used in chemical data assimilation systems (e.g. Kahnert, 2008; Benedetti et al., 2009; Liu et al., 2011). However, the new optics model we introduced here does not provide us with a linear map from the aerosol concentrations to the optical parameters. To what extent one could linearize this model and make use of its Jacobian in a data assimilation system mainly depends on the degree of nonlinearity, which would need to be investigated thoroughly.

\section{Code availability}

The SALSA aerosol microphysics code is distributed under the Apache 2.0 license, while the MATCH chemistrytransport model and the aerosol-optics database are available upon request from SMHI. 
Appendix A: Size-averaged optical properties in the external-mixture optics model

The external-mixture optics model is based on using four size bins that cover the dry-radius intervals $\left[r^{\min }, r^{\max }\right]=[0.01$, $0.05],[0.05,0.5],[0.5,1.25]$, and $[1.25,5] \mu \mathrm{m}$. The geometric mean radius $R=\sqrt{r^{\min } r^{\max }}=0.5\left(\log r^{\min }+\log r^{\max }\right)$ is given in each of these intervals by $R_{1}=0.022 \mu \mathrm{m}, R_{2}=$ $0.158 \mu \mathrm{m}, R_{3}=0.791 \mu \mathrm{m}$, and $R_{4}=2.5 \mu \mathrm{m}$. In each size bin it is assumed that the particle number density is given by a log-normal distribution

$n_{i}(r)=N_{i}^{0} /\left(\sqrt{2 \pi} r \ln \sigma_{i}\right) \exp \left[-\ln ^{2}\left(r / R_{i}\right) /\left(2 \ln ^{2} \sigma_{i}\right)\right]$,

where $\sigma_{1}=\sigma_{3}=\sigma_{4}=1.8, \sigma_{2}=1.5$ are based on measurements in Neusüß et al. (2002). Here, $N_{i}^{0}$ would be the total number density per mode if each size mode extended from $r=0$ to $r=\infty$. However, since each mode is truncated at the bin boundaries $r^{\min }$ and $r^{\max }$, the number density $N_{i}$ of particles per size bin is obtained from integration over this finite interval, i.e.

$$
N_{i}=\int_{r_{i}^{\min }}^{r_{i}^{\max }} n_{i}(r) \mathrm{d} r=N_{i}^{0} \frac{1}{2}\left[\operatorname{erf}\left(x_{i}^{\max }\right)-\operatorname{erf}\left(x_{i}^{\min }\right)\right],
$$

where $x_{i}^{\max }=\ln \left(r_{i}^{\max } / R_{i}\right) /\left(\sqrt{2} \ln \sigma_{i}\right)$, and similarly for $x_{i}^{\text {min }}$. Analogously, one obtains the particle-mass density $M_{i}$ in each size bin by integrating over the truncated log-normal mode, which yields

$$
\begin{aligned}
M_{i} & =\frac{4}{3} \pi \rho_{i} \int_{r_{i}^{\min }}^{r_{i}^{\max }} n_{i}(r) r^{3} \mathrm{~d} r \\
& =\frac{4}{3} \pi R_{i}^{3} \rho_{i} N_{i}^{0} \exp \left(\frac{9}{2} \ln ^{2} \sigma_{i}\right) \frac{1}{2}\left[\operatorname{erf}\left(y_{i}^{\max }\right)-\operatorname{erf}\left(y_{i}^{\min }\right)\right]
\end{aligned}
$$

where $y_{i}^{\max }=x_{i}^{\max }-3 \ln \sigma_{i} / \sqrt{2}$, and similarly for $y_{i}^{\min }$, and where $\rho_{i}$ is the density of the aerosol particles in the $i$ th size bin. From this we obtain the desired relation for converting the mass density $M_{i}$ into the number density $N_{i}$ :

$$
N_{i}=\frac{M_{i}}{\frac{4}{3} \pi R_{i}^{3} \rho_{i}} \cdot \frac{\operatorname{erf}\left(x_{i}^{\max }\right)-\operatorname{erf}\left(x_{i}^{\min }\right)}{\exp \left(\frac{9}{2} \ln ^{2} \sigma_{i}\right)\left[\operatorname{erf}\left(y_{i}^{\max }\right)-\operatorname{erf}\left(y_{i}^{\min }\right)\right]}
$$

Mass-mixing ratios $X_{i}$ are simply converted into mass densities $M_{i}$ according to $M_{i}=X_{i} \rho_{\text {air }}$, where $\rho_{\text {air }}$ denotes the density of air.

In the external-mixture optics database, optical properties are pre-computed by integrating optical properties at discrete sizes over the truncated log-normal size distribution. This integration is done numerically with a high size resolution. The computation is performed for different refractive indices $m$, optical wavelengths $\lambda$, and for each size bin $i$. Thus, one obtains e.g. extinction cross sections $C_{\text {ext }}(\lambda, m, i)$, which can be saved in a look-up table.

Secondary inorganic aerosols as well as organic aerosols and sea salt are assumed to be hydrophilic. We use the parameterization by Gerber (1985) to compute the wet radius $r_{\mathrm{w}}$ from the aerosol dry radius $R$, from which we obtain the volume fraction of water $f_{\mathrm{w}}=\left(r_{\mathrm{w}}^{3}-R^{3}\right) / R^{3}$. The effective refractive index $m$ of the aerosol-water mixture is computed from that of the dry aerosol, $m_{\mathrm{a}}$, and of water, $m_{\mathrm{w}}$ by use of effective-medium theory. In each grid cell, we obtain from the MATCH model, for each size bin $i$ and for each aerosol component $k$, the number density $N_{i}(k)$. From that we compute the ensemble-averaged extinction cross section

$\bar{C}_{\text {ext }}(\lambda)=\frac{1}{N} \sum_{k} \sum_{i=1}^{4} N_{i}(k) C_{\text {ext }}(\lambda, m(k), i)$,

where the total number density is given by

$N=\sum_{k} \sum_{i=1}^{4} N_{i}(k)$.

Note that the ensemble-average involves an average over both size and chemical composition. The ensemble-averaged scattering cross section $\bar{C}_{\text {sca }}(\lambda)$ is computed analogously. From this we obtain the averaged single-scattering albedo

$\bar{\omega}(\lambda)=\frac{\bar{C}_{\text {sca }}(\lambda)}{\bar{C}_{\text {ext }}(\lambda)}$.

The phase function $p(\Theta)$, and hence its first Legendre moment, known as the asymmetry parameter $g$, are normalized quantities. Here $\Theta$ denotes the scattering angle. To average these quantities, one first needs to "de-normalize" by multiplying them by the scattering cross section. Thus,

$$
\begin{gathered}
\bar{p}(\Theta ; \lambda)=\frac{1}{N \bar{C}_{\mathrm{sca}}(\lambda)} \sum_{k} \sum_{i=1}^{4} N_{i}(k) \\
C_{\mathrm{sca}}(\lambda, m(k), i) p(\Theta, m(k), i ; \lambda), \\
\bar{g}(\lambda)=\frac{1}{N \bar{C}_{\mathrm{sca}}(\lambda)} \sum_{k} \sum_{i=1}^{4} N_{i}(k) \\
C_{\mathrm{sca}}(\lambda, m(k), i) g(m(k), i ; \lambda) .
\end{gathered}
$$

Once the ensemble-averaged optical properties in each grid cell of the model domain have been computed, one can compute radiometric observables, such as the extinction aerosol optical depth

$\tau_{\text {ext }}(\lambda)=\sum_{z} N(z) \bar{C}_{\text {ext }}(\lambda, z) \Delta z$,

or the backscattering coefficient

$\beta_{\text {bak }}(\lambda, z)=\frac{1}{4 \pi} N(z) \bar{C}_{\text {sca }}(\lambda, z) \bar{p}\left(180^{\circ} ; \lambda, z\right)$,

where $z$ labels grid cells in the vertical column, and $\Delta z$ denotes the layer thickness. 


\section{Appendix B: Size-averaged optical properties in the internal-mixture model}

In SALSA the number density as a function of particle radius, $n(r)$, is given by a step function with $n_{i}(r)=$ const $_{i}$ in each size bin $i$. This makes the pre-integration of optical properties over each size bin rather simple. On the other hand, we no longer assume that all aerosol components are externally mixed. Thus the ensemble average over different chemical components $k$ is no longer given by a simple summation $\sum_{k} \cdots$, as it was in the external-mixture model. Rather, for each size bin in which several aerosol components are internally mixed one computes an effective refractive index, $m_{\text {eff }}$, by use of effective-medium theory. One then reads the optical properties for that refractive index from the lookup table. Finally, one computes ensemble-averaged optical properties by summing over all size bins, $\sum_{i} \cdots$.

\section{Appendix C: Effective-medium theory}

In effective-medium theory (EMT) one considers a composite material consisting of two materials with refractive indices $m_{1}$ and $m_{2}$ and volume fractions $f_{1}$ and $f_{2}=1-f_{1}$. One then invokes assumptions about the topology of the mixture and derives a formula for the effective refractive index, $m_{\text {eff, }}$ of the composite material. For instance, it is often the case that $f_{1} \gg f_{2}$. In this case one can regard the first material as a host matrix that contains inclusions of the second material. This is the basis of the Maxwell Garnett EMT (Maxwell Garnett, 1904). The resulting expression for $m_{\mathrm{eff}}$ is

$m_{\text {eff }}=\sqrt{m_{1}^{2} \frac{m_{1}^{2}\left(2-2 f_{2}\right)+m_{2}^{2}\left(1+2 f_{2}\right)}{m_{1}^{2}\left(2+f_{2}\right)+m_{2}^{2}\left(1-f_{2}\right)}}$.
In the Bruggemann EMT (Bruggemann, 1935) one treats both materials more symmetrically; both components are assumed to be embedded in a host matrix with an effective refractive index given by

$$
\begin{aligned}
& m_{\mathrm{eff}}=\left(\frac{1}{4} m_{1}^{2}\left(2-3 f_{2}\right)+m_{2}^{2}\left(3 f_{2}-1\right)\right. \\
& \left.+\sqrt{\frac{1}{16}\left[m_{1}^{2}\left(2-3 f_{2}\right)+m_{2}^{2}\left(3 f_{2}-1\right)\right]^{2}+\frac{1}{2} m_{1}^{2} m_{2}^{2}}\right)^{1 / 2} .
\end{aligned}
$$

Although not immediately manifest, this equation is symmetric under exchange of the two materials.

The volume fraction is obtained from the mass concentrations $M_{1}$ and $M_{2}$ computed in the transport model, i.e. $f_{2}=M_{2} /\left(M_{1}+M_{2}\right)$. In SALSA, we apply the Maxwell Garnett rule for an internal mixture of mineral-dust inclusions in a host matrix of soluble compounds. Also, in the core-greyshell model the effective refractive index of the grey shell, i.e. the homogeneous mixture of black carbon with soluble compounds, is computed with the Maxwell Garnett EMT. For mixtures of soluble compounds (sulfate, nitrate, ammonium, sea salt, organic compounds, and water) we use the Bruggemann EMT. If more than two components are mixed with each other, then the mixing rule is applied iteratively. 


\section{Appendix D: Refractive indices}

The refractive indices that are used in the new optics model (and in the effective-medium calculations) are listed in Table D1.

Table D1. Refractive index $m$ for each wavelength in the aerosol-optics look-up table and for various aerosol components.

\begin{tabular}{|c|c|c|c|c|}
\hline$\lambda(\mu \mathrm{m})$ & 000 & 2316 & 3040 & .3400 \\
\hline & $4840+0.1000 \times 10^{-7} i$ & $4840+0.1000 \times 10^{-7} i$ & $.4676+0.1000 \times 10^{-7} i$ & $1.4554+0.1000 \times 10^{-7} i$ \\
\hline$m(\mathrm{BC})$ & $0.9400+0.3500 \times 10^{0} i$ & $1.0717+0.5817 \times 10^{0} i$ & $1.3314+0.7523 \times 10^{0} i$ & $1.4471+0.7214 \times 10^{0} i$ \\
\hline$m(\mathrm{OC})$ & $.5300+0.5000 \times 10^{-2} i$ & $1.5300+0.5000 \times 10^{-2} i$ & $1.5300+0.5000 \times 10^{-2} i$ & $1.5300+0.5000 \times 10^{-2} i$ \\
\hline$\imath(\mathrm{NaCl})$ & $5100+0.5000 \times 10^{-5} i$ & $1.5100+0.5000 \times 10^{-5} i$ & $1.5100+0.1866 \times 10^{-5} i$ & $1.5100+0.6592 \times 10^{-6} i$ \\
\hline$m$ (Dust) & $190+0.2070 \times 10^{-1} i$ & $2070 \times 10^{-1} i$ & $1.5240+0.1947 \times 10^{-1} i$ & $1.5272+0.1683 \times 10^{-1} i$ \\
\hline$m\left(\mathrm{H}_{2} \mathrm{O}\right)$ & $1.4517+0.1101 \times 10^{-6} i$ & $1.4094+$ & $1.3701+0.3879 \times 10^{-8} i$ & $1.3604+0.2758 \times 10^{-8} i$ \\
\hline m) & 550 & 3800 & 3932 & 4400 \\
\hline & & $1000 \times 10^{-7} i$ & $.4416+0.1000 \times 10^{-7} i$ & $1.4336+0.1000 \times 10^{-7} i$ \\
\hline $\mathrm{BC})$ & & $.5757+0.6871 \times 10^{0} i$ & $.6181+0.6758 \times 10^{0} i$ & $1.6771+$ \\
\hline$m(\mathrm{OC})$ & )$^{-2} i$ & $\times 10^{-2} i$ & $5000 \times 10^{-2} i$ & $1.5300+0.5000 \times 10^{-2} i$ \\
\hline$m(\mathrm{NaCl})$ & & & $998 \times 10^{-7} i$ & $0^{-7} i$ \\
\hline$m$ (Dust) & ${ }^{-1} i$ & $0 \times 10^{-2} i$ & $5147+0.2170 \times 10^{-2} i$ & $1.5135+0.1400 \times 10^{-2} i$ \\
\hline$m\left(\mathrm{H}_{2} \mathrm{O}\right)$ & & & & \\
\hline & & & & \\
\hline & $-7 i$ & $-7 i$ & $0^{-7} i$ & $0^{-7} i$ \\
\hline & & & & $1.8097+$ \\
\hline & & & $10^{-2} i$ & 1.5300 \\
\hline$m(\mathrm{NaCl})$ & & ${ }^{-7} i$ & $1.5000+$ & $1.4900+$ \\
\hline ust) & & & $-2 i$ & $10^{-3} i$ \\
\hline$m\left(\mathrm{H}_{2} \mathrm{O}\right)$ & $1.3394+0$ & $1.3371+0$ & $1.3370+0$ & $1.3297+$ \\
\hline (ump & & & & \\
\hline$m \mathrm{r}$ & $-7 i$ & $-6 i$ & ${ }^{-5} i$ & $0^{-5} i$ \\
\hline & & & & $10^{0} i$ \\
\hline l(OC) & & $-2 i$ & & $10^{-1} i$ \\
\hline$m(\mathrm{~N}$ & $-6 i$ & $10^{-4} i$ & $1.4700+$ & $1.4700+$ \\
\hline & & $0 \times 10^{-3} i$ & $1.5190+$ & $1.5190+$ \\
\hline$m\left(\mathrm{H}_{2} \mathrm{O}\right)$ & $1.3287+0$ & $1.3243+0$ & $1.3215+c$ & $1.3213+$ \\
\hline & & & & \\
\hline S & & & $10^{-3} i$ & $1.3926+$ \\
\hline (BC) & & & & $10^{0} i$ \\
\hline & & & & $10^{-1} i$ \\
\hline$m(\mathrm{I}$ & & $10^{-3} i$ & $10^{-3} i$ & $10^{-3} i$ \\
\hline (Dust) & $\times 10^{-3} i$ & $1.5188+0.6$ & $\times 10^{-3} i$ & $1.5180+0.9990 \times 10^{-3} i$ \\
\hline$m\left(\mathrm{H}_{2} \mathrm{O}\right)$ & & & & $10^{-3} i$ \\
\hline & & & & \\
\hline & & & & $0^{0} i$ \\
\hline & 2.0 & 2.051 & $2.1099+c$ & $2.1955+0.1088 \times 10^{1} i$ \\
\hline 7) & & $1.4554+0.9641 \times 10^{-2} i$ & $\times 10^{-2} i$ & $1.4800+0.7000 \times 10^{-2} i$ \\
\hline$m($ & & & $-2 i$ & $\times 10^{-2} i$ \\
\hline$m$ (Dust) & & $-2 i$ & $1.5180+$ & $1.5180+0.2805 \times 10^{-1} i$ \\
\hline$m\left(\mathrm{H}_{2} \mathrm{O}\right)$ & 1.294 & 1.275 & $1.1278+$ & $1.3913+0.1237 \times 10^{-1} i$ \\
\hline & & & & \\
\hline & $i$ & & & $0^{0} i$ \\
\hline & 2.2 & $2.6572+0$ & $2.9285+0$ & $3.0719+0.2$ \\
\hline () & $1.4800+$ & $\times 10^{-1} i$ & $1.7600+0.7000 \times 10^{-1} i$ & $1.6352+0.5117 \times 10^{-1} i$ \\
\hline$m($ & 1. & $581 \times 10^{-1} i$ & $0 \times 10^{-1} i$ & $10^{-1} i$ \\
\hline & & $1.1798+0.1015 \times 10^{0} i$ & $1.9100+0.2500 \times 10^{0} i$ & $1.7614+0.4543 \times 10^{0} i$ \\
\hline$m\left(\mathrm{H}_{2} \mathrm{O}\right)$ & $1.3840+0$ & $1.2676+0.3436 \times 10^{-1} i$ & $1.1531+0.7145 \times 10^{-1} i$ & $1.0874+0.2243 \times 10^{0} i$ \\
\hline
\end{tabular}




\section{The Supplement related to this article is available online at doi:10.5194/gmd-9-1803-2016-supplement.}

Acknowledgements. E. Andersson acknowledges funding from the Swedish National Space Board within the OSCES project (no. 101/13). M. Kahnert has been funded by the Swedish Research Council (Vetenskapsrådet) within the AGES project (project 621-2011-3346).

Edited by: S. Unterstrasser

\section{References}

Adachi, K. and Buseck, P. R.: Internally mixed soot, sulfates, and organic matter in aerosol particles from Mexico City, Atmos. Chem. Phys., 8, 6469-6481, doi:10.5194/acp-8-6469-2008, 2008.

Adachi, K., Chung, S. H., Friedrich, H., and Buseck, P. R.: Fractal parameters of individual soot particles determined using electron tomography: Implications for optical properties, J. Geophys. Res., 112, D14202, doi:10.1029/2006JD008296, 2007.

Andersson, C., Langner, J., and Bergström, R.: Interannual variation and trends in air pollution over Europe due to climate variability during 1958-2001 simulated with a regional CTM coupled to the ERA40 reanalysis, Tellus B, 59, 77-98, 2007.

Andersson, C., Bergström, R., Bennet, C, Thomas, M., Robertson, L., Kokkola, H., Korhonen, H., and Lehtinen, K.: MATCH-SALSA - Multi-scale Atmospheric Transport and Chemistry model coupled to the SALSA aerosol microphysics model, SMHI Report RMK 115, available at: http://www.smhi.se/publikationer/match-salsa-multi-scaleatmospheric-transport-and-chemistry-model-coupled-to-thesalsa-aerosol-microphysics-model-1.34623 (last access: 10 February 2016), 2013.

Andersson, C., Bergström, R., Bennet, C., Robertson, L., Thomas, M., Korhonen, H., Lehtinen, K. E. J., and Kokkola, H.: MATCHSALSA - Multi-scale Atmospheric Transport and CHemistry model coupled to the SALSA aerosol microphysics model Part 1: Model description and evaluation, Geosci. Model Dev., 8, 171-189, doi:10.5194/gmd-8-171-2015, 2015.

Benedetti, A., Morcrette, M. J.-J., Boucher, O., Dethof, A., Engelen, R. J., Huneeus, M. F. H. F. N., Jones, L., Kinne, J. W. K., Mangold, A., Razinger, M., Simmons, A. J., and Suttie, M.: Aerosol analysis and forecast in the European Centre for Medium-Range Weather Forecasts Integrated Forecast System: 2. Data assimilation, J. Geophys. Res., 114, D13205, doi:10.1029/2008JD011115, 2009.

Bond, T. C. and Bergstrom, R. W.: Light absorption by carbonaceous particles: An investigative review, Aerosol Sci. Tech., 40, 27-67, 2006.

Bruggemann, D. A. G.: Berechnung verschiedener physikalischer Konstanten von heterogenen Substanzen. 1. Dielektrizitätskonstanten und Leitfähigkeiten der Mischkörper aus isotropen Substanzen, Ann. Phys.-Berlin, 24, 636-664, 1935.
Chakrabarty, R. K., Beres, N. D., Moosmüller, H., China, S., Mazzoleni, C., Dubey, M. K., Liu, L., and Mishchenko, M. I.: Soot superaggregates from flaming wildfires and their direct radiative forcing, Scientific Reports, 4, 5508 EP, doi:10.1038/srep05508, 2014.

Chang, H. and Charalampopoulos, T. T.: Determination of the wavelength dependence of refractive indices of flame soot, P. R. Soc. A, 430, 577-591, 1990.

China, S., Salvadori, N., and Mazzoleni, C.: Effect of Traffic and Driving Characteristics on Morphology of Atmospheric Soot Particles at Freeway On-Ramps, Environ. Sci. Technol., 48, 3128-3135, doi:10.1021/es405178n, 2014.

Dubovik, O., Sinyuk, A., Lapyonok, T., Holben, B. N., Mishchenko, M. I., Yang, P., Eck, T. F., Volten, H., Muñoz, O., Veihelmann, B., van der Zande, W. J., Leon, J.-F., Sorokin, M., and Slutsker, I.: Application of spheroid models to account for aerosol particle nonsphericity in remote sensing of desert dust, J. Geophys. Res., 111, D11208, doi:10.1029/2005JD006619, 2006.

Foltescu, V., Pryor, S. C., and Bennet, C.: Sea salt generation, dispersion and removal on the regional scale, Atmos. Environ., 39 , 2123-2133, 2005

Gerber, H. E.: Relative-humidity parameterization of the Navy Aerosol Model (NAM), Tech. Rep. 8956, Naval Research Laboratory, Washington, DC, 1985.

Jacobson, M. Z.: A physically-based treatment of elemental carbon optics: Implications for global direct forcing of aerosols, Geophys. Res. Lett, 27, 217-220, 2000.

Jones, A. R.: Light scattering in combustion, in: Light Scattering Reviews, edited by: Kokhanovsky, A., Springer, Berlin, 393-444, 2006.

Kahnert, F. M.: Reproducing the optical properties of fine desert dust aerosols using ensembles of simple model particles, J. Quant. Spectrosc. Ra., 85, 231-249, 2004.

Kahnert, M.: Variational data analysis of aerosol species in a regional CTM: background error covariance constraint and aerosol optical observation operators, Tellus B, 60, 753-770, 2008.

Kahnert, M.: Modelling the optical and radiative properties of freshly emitted light absorbing carbon within an atmospheric chemical transport model, Atmos. Chem. Phys., 10, 1403-1416, doi:10.5194/acp-10-1403-2010, 2010a.

Kahnert, M.: On the discrepancy between modelled and measured mass absorption cross sections of light absorbing carbon aerosols, Aerosol Sci. Tech., 44, 453-460, 2010b.

Kahnert, M. and Devasthale, A.: Black carbon fractal morphology and short-wave radiative impact: a modelling study, Atmos. Chem. Phys., 11, 11745-11759, doi:10.5194/acp-11-117452011, 2011.

Kahnert, M., Nousiainen, T., and Lindqvist, H.: Models for integrated and differential scattering optical properties of encapsulated light absorbing carbon aggregates, Opt. Express, 21, 7974 7993, 2013.

Kahnert, M., Nousiainen, T., and Lindqvist, H.: Review: Model particles in atmospheric optics, J. Quant. Spectrosc. Ra., 146, 41-58, 2014.

Khlebtsov, N. G.: Orientational averaging of light-scattering observables in the T-matrix approach, Appl. Optics, 31, 5359-5365, 1992.

Klingmüller, K., Steil, B., Brühl, C., Tost, H., and Lelieveld, J.: Sensitivity of aerosol radiative effects to different mixing assump- 
tions in the AEROPT 1.0 submodel of the EMAC atmosphericchemistry-climate model, Geosci. Model Dev., 7, 2503-2516, doi:10.5194/gmd-7-2503-2014, 2014.

Kokkola, H., Korhonen, H., Lehtinen, K. E. J., Makkonen, R., Asmi, A., Järvenoja, S., Anttila, T., Partanen, A.-I., Kulmala, M., Järvinen, H., Laaksonen, A., and Kerminen, V.-M.: SALSA - a Sectional Aerosol module for Large Scale Applications, Atmos. Chem. Phys., 8, 2469-2483, doi:10.5194/acp-8-2469-2008, 2008.

Kupiainen, K. and Klimont, Z.: Primary emissions of submicron and carbonaceaous particles in Europe and the potential for their control, Tech. Rep. IR-04-079, IIASA, Laxenburg, Austria, 2004.

Kupiainen, K. and Klimont, Z.: Primary emissions of fine carbonaceous particles in Europe, Atmos. Environ., 41, 2156-2170, 2007.

Kylling, A., Bais, A. F., Blumthaler, M., Schreder, J., Zerefos, C. S., and Kosmidis, E.: The effect of aerosols on solar UV irradiances during the photochemical activity and solar ultraviolet radiation campaign, J. Geophys. Res., 103, 26051-26060, 1998.

Liu, Z., Liu, Q., Lin, H.-C., Schwartz, C. S., Lee, Y.-H., and Wang, T.: Three-dimensional variational assimilation of MODIS aerosol optical depth: Implementation and application to a dust storm over East Asia, J. Geophys. Res., 116, D23206, doi:10.1029/2011JD016159, 2011.

Mackowski, D. W. and Mishchenko, M. I.: A multiple sphere Tmatrix Fortran code for use on parallel computer clusters, J. Quant. Spectrosc. Ra., 112, 2182-2192, 2011.

Mårtensson, E. M., Nilsson, E. D., de Leeuw, G., Cohen, L. H., and Hansson, H.-C.: Laboratory simulations and parameterization of the primary marine aerosol production, J. Geophys. Res., 108, 4297, doi:10.1029/2002JD002263, 2003.

Matsui, H., Koike, M., Kondo, Y., Moteki, N., Fast, J. D., and Zaveri, R. A.: Development and validation of a black carbon mixing state resolved three-dimensional model: Ageing processes and radiative impact, J. Geophys. Res., 118, 2304-2326, doi:10.1029/2012JD018446, 2013.

Maxwell Garnett, J. C.: Colours in metal glasses and in metallic films, Philos. T. R. Soc. A, 203, 385-420, 1904.

Mishchenko, M. I., Travis, L. D., and Lacis, A. A.: Scattering, Absorption, and Emission of Light by Small Particles, Cambridge University Press, Cambridge, 2002.

Monahan, E. C., Spiel, D. E., and Davidson, K. L.: A model of marine aerosol generation via whitecaps and wave disruption, in: Oceanic Whitecaps and their Role in Air-Sea Exchange, edited by: Monahan, E. C. and Niocaill, G. M., D Reidel, Norwell, MA, 167-174, 1986.
Neusüß, C., Wex, H., Birmili, W., Wiedensohler, A., Koziar, C., Busch, B., Brüggemann, E., Gnauk, T., Ebert, M., and Covert, D. S.: Characterization and parameterization of atmospheric particle number-, mass-, and chemical-size distributions in central Europe during LACE 98 and MINT, J. Geophys. Res., 107, LAC 9-1-LAC 9-13, doi:10.1029/2001JD000514, 2002.

Nousiainen, T.: Optical modeling of mineral dust particles: a review, J. Quant. Spectrosc. Ra., 110, 1261-1279, 2009.

Nousiainen, T., Kahnert, M., and Veihelmann, B.: Light scattering modeling of small feldspar aerosol particles using polyhedral prisms and spheroids, J. Quant. Spectrosc. Ra., 101, 471-487, 2006.

Scarnato, B. V., Vahidinia, S., Richard, D. T., and Kirchstetter, T. W.: Effects of internal mixing and aggregate morphology on optical properties of black carbon using a discrete dipole approximation model, Atmos. Chem. Phys., 13, 5089-5101, doi:10.5194/acp-13-5089-2013, 2013.

Scarnato, B. V., China, S., Nielsen, K., and Mazzoleni, C.: Perturbations of the optical properties of mineral dust particles by mixing with black carbon: a numerical simulation study, Atmos. Chem. Phys., 15, 6913-6928, doi:10.5194/acp-15-6913-2015, 2015.

Robertson, L., Langner, J., and Engardt, M.: An Eulerian limitedarea atmospheric transport model, J. Appl. Meteorol., 38, 190210, 1999.

Saide, P. E., Carmichael, G. R., Liu, Z., Schwartz, C. S., Lin, H. C., da Silva, A. M., and Hyer, E.: Aerosol optical depth assimilation for a size-resolved sectional model: impacts of observationally constrained, multi-wavelength and fine mode retrievals on regional scale analyses and forecasts, Atmos. Chem. Phys., 13, 10425-10444, doi:10.5194/acp-13-10425-2013, 2013.

Undén, P., Rontu, L., Järvinen, H., Lynch, P., Calvo, J., Cats, G., Cuxart, J., Eerola, K., Fortelius, C., Garcia-Moya, J. A., Jones, C., Lenderlink, G., McDonald, A., McGrath, R., Navascues, B., Nielsen, N. W., Ødegaard, V., Rodriguez, E., Rummukainen, M., Rõõm, R., Sattler, K., Hansen Sass, B., Savijärvi, H., Schreur, B. W., Sigg, R., The, H., and Tijm, A.: HIRLAM-5 Scientic Documentation, available at: http://www.hirlam.org/index.php/ documentation (last access: 15 February 2016), 2002.

Yurkin, M. A. and Hoekstra, A. G.: The discrete dipole approximation: an overview and recent developments, J. Quant. Spectrosc. Ra., 106, 558-589, 2007. 